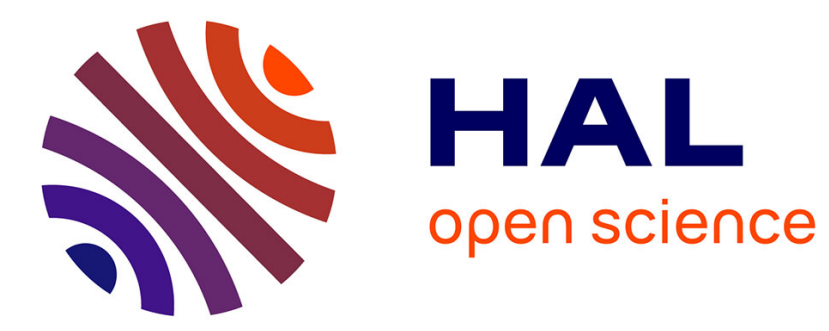

\title{
Multiple Light Scattering in Ordered Nematic Liquid Crystals
}

Anne Heiderich, Roger Maynard, B. van Tiggelen

\section{To cite this version:}

Anne Heiderich, Roger Maynard, B. van Tiggelen. Multiple Light Scattering in Ordered Nematic Liquid Crystals. Journal de Physique II, 1997, 7 (5), pp.765-792. 10.1051/jp2:1997104 . jpa-00248477

\section{HAL Id: jpa-00248477 https://hal.science/jpa-00248477}

Submitted on 1 Jan 1997

HAL is a multi-disciplinary open access archive for the deposit and dissemination of scientific research documents, whether they are published or not. The documents may come from teaching and research institutions in France or abroad, or from public or private research centers.
L'archive ouverte pluridisciplinaire HAL, est destinée au dépôt et à la diffusion de documents scientifiques de niveau recherche, publiés ou non, émanant des établissements d'enseignement et de recherche français ou étrangers, des laboratoires publics ou privés. 


\title{
Multiple Light Scattering in Ordered Nematic Liquid Crystals
}

\author{
Anne Heiderich, Roger Maynard and Bart A. van Tiggelen $\left({ }^{*}\right)$ \\ Laboratoire de Physique et Modélisation des Systèmes Condensés, CNRS, \\ Maison des Magistères, BP. 166, 38042 Grenoble Cedex 9, France
}

(Received 10 June 1996, received in final form 19 December 1996, accepted 3 February 1997)

\author{
PACS.42.25.Lc - Birefringence \\ PACS.61.30.Gd - Orientational order of liquid crystals; electric and magnetic field effects \\ on order
}

\begin{abstract}
We study multiple light scattering in ordered nematic liquid crystals from longrange dielectric fluctuations. The mass operator is calculated in the first Born approximation of Dyson's equation for the average amplitude. New results are found concerning the spectral function. In addition, the average intensity is obtained numerically using a Monte-Carlo simulation. We simulated the anisotropic shape of the coherent backscattering cone, the time-of-flight distribution functions and the polarization in the diffusive regime.
\end{abstract}

\section{Introduction}

Multiple scattering of light and related phenomena like coherent backscattering have been studied for all kinds of disordered materials such as isotropic [1,2], Faraday active [3-5], amplifying [6] and nonlinear media [7].

In two recent papers $[8,9]$ we studied light propagation in an anisotropic medium, i.e. an ordered nematic liquid crystal, taking into account long-range dielectric fluctuations. Since nematic liquid crystals possess an anisotropic polarizability, any distortion of the molecular orientation, for example due to thermal fluctuations, will cause scattering or even multiple scattering of light. Those long-range fluctuations are of particular interest. Few experimental $[10,11]$ and theoretical [12] studies have been done. Recently, we employed a scalar approximation, thereby neglecting all polarization effects, in order to investigate the influence of long-range dielectric fluctuations on light propagation [9]. Interesting results concerning the spectral distribution of the transport modes were obtained. Results of the analytic vector theory have already been published in reference [8] and independently by Stark and Lubensky [13]. They seem to agree with the recent first experiments [14].

In this article we present a vector treatment of the radiative transfer in these materials, which takes polarization correctly into account. The average field amplitude is obtained analytically solving Dyson's equation while a numerical simulation has been employed to determine the average intensity. The latter approach corresponds to the solution of the Bethe-Salpeter equation using Boltzmann's approximation, without relying upon the diffusion approximation.

$\left({ }^{*}\right)$ Author for correspondence (e-mail: tiggelen@mgstsrv.polycnrs-gre.fr)

(C) Les Éditions de Physique 1997 
This paper is structured in the following way: first, we briefly recall the characteristics of light propagation in anisotropic media such as birefringence. The correlation function describing the thermal fluctuations of the molecular orientation is reviewed. We solve Dyson's equation, i.e. we determine the change of refractive index and the scattering mean free path. The spectral function will be discussed and a comparison made with the anomalous line shape predicted within the scalar approximation. An expression for the average amplitude Green's function is given explicitly.

In the second part, we present a Monte-Carlo algorithm used in order to obtain the average intensity numerically. This Monte-Carlo method generalizes previous algorithms in that it treats four random variables. The results are presented in the last section. We analyze the line shape of the coherent backscattering cone, investigate the time-of-flight distribution functions and the polarization of multiply scattered light. The transport mean free path is obtained for several values of the anisotropy of the material.

\section{Light Propagation in Ordered Nematics}

One of the most important effects observed in media with an anisotropic dielectric tensor is birefringence. At an interface, the incident plane wave is decomposed into two propagating eigenstates of orthogonal polarization, corresponding to two propagation eigenmodes of the anisotropic medium. They are known as the ordinary ray $|\mathbf{o}\rangle$ and extraordinary ray $|\mathbf{e}\rangle$. A third eigenstate, the longitudinal wave $|\mathbf{p}\rangle=\hat{\mathbf{p}}$, does not propagate and is therefore generally neglected. However, the longitudinal wave plays an important role for the correction of the refractive index of the medium [15].

The polarization vector of the ordinary ray is located perpendicular to the direction of propagation $\mathbf{p}$ and the optical axis of the medium $\mathbf{n}_{0}$. It is a transverse mode as normally encountered in an isotropic medium. Moreover, its dispersion law is isotropic, this being the reason why it is called "ordinary". On the contrary, the extraordinary ray is not a transverse mode. Its polarization lies in the plane formed by the wave vector and the optical axis. In addition, its dispersion law is anisotropic, i.e. the index of refraction of the extraordinary ray depends on the angle formed between the direction of propagation and the optical axis. Explicitly, the dispersion laws and unit vectors of the two polarization are given by

$$
\begin{array}{cc}
\text { ordinary ray } & p_{e}=\sqrt{\frac{\text { extraordinary ray }}{\varepsilon_{\|} \cos ^{2} \theta+\varepsilon_{\perp} \sin ^{2} \theta}} \frac{\omega}{c} \equiv n_{e} \frac{\omega}{c} \\
|\mathbf{s}\rangle=\frac{\hat{\mathbf{p}} \times \mathbf{n}}{|\hat{\mathbf{p}} \times \mathbf{n}|} ; & |\mathbf{e}\rangle=\frac{\varepsilon_{\|}\langle\mathbf{p} \mid \mathbf{n}\rangle \hat{\mathbf{p}}-\left\langle\mathbf{p}\left|\varepsilon_{0}\right| \mathbf{p}\right\rangle \mathbf{n}}{|\hat{\mathbf{p}} \times \mathbf{n}| \sqrt{\left\langle\mathbf{p}\left|\varepsilon_{0}^{2}\right| \mathbf{p}\right\rangle}}
\end{array}
$$

It is important to note that, except parallel to the optical axis, no elliptical polarization can exist in an anisotropic medium because different wave vectors correspond to the two different eigenmodes. Therefore, a constant phase difference between the two linear polarizations cannot be maintained over a distance longer than the wavelength [16].

2.1. Unperturbed Green's Function. - If thermal fluctuations of the director are ignored, the transport of light is described by the Helmholtz equation

$$
\left[\frac{\omega^{2}}{c^{2}} \varepsilon_{0}-p^{2}\left(\mathbf{1}-\hat{\mathbf{p}} \hat{\mathbf{p}}^{\mathrm{T}}\right)\right] \mathbf{G}_{0}(\mathbf{p}, \omega)=\mathbf{1}
$$


Its solution, the unperturbed Green's function $\mathbf{G}_{0}(\mathbf{p}, \omega)$ is a second rank tensor. It is diagonal with respect to the non-orthogonal basis of the transport eigenmodes $|\mathbf{o}\rangle,|\mathbf{e}\rangle$ and the longitudinal mode $|\mathbf{p}\rangle$ and reads

$$
\begin{aligned}
\mathbf{G}_{0}(\mathbf{p}, \omega) & =\sum_{\jmath \in\{o, e, p\}} g_{\jmath}|\mathbf{j}\rangle\langle\mathbf{j}| \\
& =\frac{|\mathbf{o}\rangle\langle\mathbf{o}|}{\left(p_{o}+i 0^{+}\right)^{2}-p^{2}}+\frac{1}{\cos ^{2} \delta_{e}} \frac{|\mathbf{e}\rangle\langle\mathbf{e}|}{\left(p_{e}+i 0^{+}\right)^{2}-p^{2}}+\frac{|\mathbf{p}\rangle\langle\mathbf{p}|}{\left(\omega^{2} / c^{2}\right)\left\langle\mathbf{p}\left|\varepsilon_{0}\right| \mathbf{p}\right\rangle}
\end{aligned}
$$

The extrordinary polarization is not orthogonal to the direction of propagation, the displacement field rather than the electrical field being perpendicular to $\mathbf{p}$. The difference between those two directions depends on the angle formed by the optical axis and the direction of propagation and its cosine is given by

$$
\cos \delta_{e}=\frac{\left\langle\mathbf{p}\left|\varepsilon_{0}\right| \mathbf{p}\right\rangle}{\sqrt{\left\langle\mathbf{p}\left|\varepsilon_{0}^{2}\right| \mathbf{p}\right\rangle}} .
$$

This angle equals the angle between the group velocity and the phase velocity of the extraordinary ray.

2.2. Correlation Function. - When thermal fluctuations of the molecular orientations are present, the local director $\mathbf{n}$ differs from the average value $\mathbf{n}_{0}$ by a deviation $\delta \mathbf{n}$. The dielectric tensor of the medium is given by

$$
\varepsilon=\varepsilon_{\perp} \mathbf{1}+\varepsilon_{\mathrm{a}} \mathbf{n n}^{\mathrm{T}}=\varepsilon_{0}+\varepsilon_{\mathrm{a}}\left[\delta \mathbf{n n}_{0}^{\mathrm{T}}+\mathbf{n}_{0} \delta \mathbf{n}^{\mathrm{T}}\right]+\mathcal{O}\left(\delta n^{2}\right)
$$

where $\varepsilon_{\mathrm{a}}=\varepsilon_{\|}-\varepsilon_{\perp}$ describes the anisotropy of the medium. The induced fluctuations of the dielectric constant $\varepsilon=\varepsilon_{0}+\delta \varepsilon$ are given by [17]:

$$
\delta \varepsilon=\varepsilon_{\mathrm{a}} \sum_{\alpha=1}^{2} \delta n_{\alpha}\left[\mathbf{e}_{\alpha} \mathbf{n}_{0}^{\mathrm{T}}+\mathbf{n}_{0} \mathbf{e}_{\alpha}^{\mathrm{T}}\right]+\mathcal{O}\left(\delta \mathbf{n}^{2}\right)
$$

The unit vectors $\mathbf{e}_{\alpha}$,

$$
\mathbf{e}_{1}=\mathbf{e}_{2} \times \mathbf{n} \text { and } \mathbf{e}_{2}=\frac{\mathbf{q} \times \mathbf{n}}{|\mathbf{q} \times \mathbf{n}|}
$$

are the orthogonal eigenmodes of the deviation $\delta \mathbf{n}$. The scattering vector $\mathbf{q}=\mathbf{k}-\mathbf{p}$ represents the difference between the wave vector of the scattered wave $\mathbf{k}$ and the incident direction of propagation $\mathbf{p}$.

A striking effect is observed concerning the ordinary polarization $[17,18]$. An incident wave with ordinary polarization has to change its state of polarization during a collision. Mathematically, this means $\langle\mathbf{o}|\delta \varepsilon| \mathbf{o}\rangle=0$. The reason for this surprising selection rule is, that to first order in $\delta n$, the dielectric function in the plane perpendicular to the optical axis is not affected. By choosing the ordinary polarization state for the incident as well as for the scattered wave, we restrict ourselves to this $2 \mathrm{D}$ subspace. Therefore, no scattering is observed in the $o \rightarrow o$ channel. For the same reason, an extraordinary ray propagating parallel to the optical axis will never be scattered in the forward direction.

An explicit expression for the two-point correlation function $\boldsymbol{\Gamma}(\mathbf{q})$, which is a fourth rank tensor describing the tensor aspect of the interaction, has been derived by de Gennes [17]:

$$
\begin{aligned}
\boldsymbol{\Gamma}(\mathbf{q}) & =\langle\delta \varepsilon(\mathbf{q}) \delta \varepsilon(0)\rangle \\
& =\frac{\varepsilon_{\perp}^{2} \Lambda}{q^{2}+1 / \xi^{2}} \sum_{\alpha=1}^{2}\left[\mathbf{n}_{0} \mathbf{e}_{\alpha}^{\mathrm{T}}+\mathbf{e}_{\alpha} \mathbf{n}_{0}^{\mathrm{T}}\right] \otimes\left[\mathbf{n}_{0} \mathbf{e}_{\alpha}^{\mathrm{T}}+\mathbf{e}_{\alpha} \mathbf{n}_{0}^{\mathrm{T}}\right]
\end{aligned}
$$


The coupling parameter $\Lambda$ is rather small, $\omega / c \Lambda \sim 10^{-4}-10^{-3}$, depending on the particular choice of the material, i.e. its dielectric anisotropy $\varepsilon_{\mathrm{a}}$ and its elastic constant $K_{3}$. Moreover, $\Lambda$ varies linearly with temperature. It is about one order of magnitude larger than the typical molecular size $a_{0}$.

In real space the elastic deformations are of long range $[17,18]$. In fact, they vary typically like $1 / r$ eventually attenuated by an exponential $\exp (-r / \xi)$. The correlation length $\xi$ of the fluctuations is given by

$$
\xi=\sqrt{\frac{K_{3}}{\chi_{\mathrm{a}} H^{2}}}
$$

where $K_{3}$ is the elastic constant. For a vanishing magnetic field, the correlation length is only limited by the sample size in the case of oriented nematics, or by the size of the domains which exist in an non-oriented liquid crystal.

\section{Mass Operator}

In this section we calculate the average field in a nematic liquid crystal when thermal fluctuations of the molecular orientations are present. We have to solve Dyson's equation. The average field propagator can formally be written as:

$$
\langle\mathbf{G}(\mathbf{p}, \omega)\rangle=\frac{1}{\frac{\omega^{2}}{c^{2}} \varepsilon_{0}-p^{2}\left(\mathbf{1}-\hat{\mathbf{p}} \hat{\mathbf{p}}^{\mathrm{T}}\right)-\mathbf{\Sigma}(\mathbf{p}, \omega)}
$$

The real difficulty consists in calculating the mass operator $\boldsymbol{\Sigma}(\mathbf{p}, \omega)$. We will do this in the first Born approximation. This approximation consists in neglecting all corrections to the mass operator which are of higher order in the density. It is justified because of the smallness of the coupling parameter $\Lambda$. The mass operator is given by the convolution of the two-point correlation function $\boldsymbol{\Gamma}$ with the unperturbed Green's function $\mathbf{G}_{0}$ [12]:

$$
\begin{aligned}
(2 \pi)^{3} \boldsymbol{\Sigma}(\mathbf{p}, \omega) \delta\left(\mathbf{p}-\mathbf{p}^{\prime}\right) & =\ldots+ \\
\Rightarrow \boldsymbol{\Sigma}(\mathbf{p}, \omega) & \approx \frac{z^{4}}{(2 \pi)^{3}} \int \boldsymbol{\Gamma}(\mathbf{p}-\mathbf{k}) \cdot \mathbf{G}_{0}(\mathbf{k}, \omega) \mathrm{d} \mathbf{k}
\end{aligned}
$$

where we have used the notation $z=\omega / c_{0}, c_{0}$ being the velocity of light in the vacuum while $c$ denotes the velocity of the ordinary ray. More explicitly, we have to solve the following integrals:

$$
\begin{aligned}
\mathbf{\Sigma}(\mathbf{p}, \omega)= & \frac{(\omega / c)^{4}}{(2 \pi)^{3}} \Lambda\left\{\int \mathrm{d} \mathbf{k} \sum_{j=o}^{e} \sum_{\alpha=1}^{2} \frac{1}{\cos ^{2} \delta_{j}} \frac{c^{2}}{p_{j}^{2}-p^{2}+i 0^{+}}\right. \\
& \times \frac{[|\mathbf{n}\rangle\langle\boldsymbol{\alpha} \mid \mathbf{j}\rangle+|\boldsymbol{\alpha}\rangle\langle\mathbf{n} \mid \mathbf{j}\rangle] \otimes[\langle\boldsymbol{\alpha} \mid \mathbf{j}\rangle\langle\mathbf{n}|+\langle\mathbf{n} \mid \mathbf{j}\rangle\langle\boldsymbol{\alpha}|]}{\left\langle\mathbf{q}_{\jmath} \mid \mathbf{n}\right\rangle^{2}+A\left[q_{j}^{2}-\left\langle\mathbf{q}_{\jmath} \mid \mathbf{n}\right\rangle^{2}\right]+1 / \xi^{2}} \\
& \left.+\frac{c^{2}}{\omega^{2}} \int \mathrm{d} \mathbf{k} \sum_{\alpha=1}^{2} \frac{1}{\left\langle\mathbf{k}\left|\varepsilon_{0}\right| \mathbf{k}\right\rangle} \frac{[|\mathbf{n}\rangle\langle\boldsymbol{\alpha} \mid \mathbf{k}\rangle+|\boldsymbol{\alpha}\rangle\langle\mathbf{n} \mid \mathbf{k}\rangle] \otimes[\langle\boldsymbol{\alpha} \mid \mathbf{k}\rangle\langle\mathbf{n}|+\langle\mathbf{n} \mid \mathbf{k}\rangle\langle\boldsymbol{\alpha}|]}{\left\langle\mathbf{q}_{\jmath} \mid \mathbf{n}\right\rangle^{2}+A\left[q_{j}^{2}-\left\langle\mathbf{q}_{\jmath} \mid \mathbf{n}\right\rangle^{2}\right]+1 / \xi^{2}}\right\}
\end{aligned}
$$

where $\mathbf{q}_{\jmath}=\mathbf{k}_{\boldsymbol{j}}-\mathbf{p}$ is the scattering vector, $|\mathbf{n}\rangle$ indicates the optical axis and $|\alpha\rangle=\mathbf{e}_{\alpha}(\alpha=1,2)$ are the unit vectors given by equation ( 7 ) of the fluctuation eigenmodes. The detailed angular dependence of the numerator is shown in Table I. Some interesting effects can be deduced qualitatively from equation (13). 
Table I. - The angular dependence of the numerator in the integral (13).

\begin{tabular}{|c|c|c|}
\hline$i \rightarrow f$ & $j$ & $Q_{\imath f}^{\jmath}=\sum_{\alpha=1}^{2}[\langle\mathbf{f} \mid \mathbf{n}\rangle\langle\alpha \mid \mathbf{j}\rangle+\langle\mathbf{f} \mid \alpha\rangle\langle\mathbf{n} \mid \mathbf{j}\rangle][\langle\alpha \mid \mathbf{j}\rangle\langle\mathbf{n} \mid \mathbf{i}\rangle+\langle\mathbf{n} \mid \mathbf{j}\rangle\langle\alpha \mid \mathbf{i}\rangle]$ \\
\hline$(o \rightarrow o)$ & $k$ & $\begin{array}{c}\frac{\sin ^{2} \theta}{\varepsilon^{2} \cos ^{2} \theta+\sin ^{2} \theta} \\
\cos ^{2} \theta\end{array}$ \\
\hline $\begin{array}{l}(o \rightarrow e) \\
\text { et } \\
(e \rightarrow o)\end{array}$ & $o$ & $\begin{array}{c}-\frac{\varepsilon \sin \theta \cos \theta \sin \vartheta \sin \phi}{\left(\varepsilon^{2} \cos ^{2} \theta+\sin ^{2} \theta\right) \sqrt{\varepsilon^{2} \cos ^{2} \vartheta+\sin ^{2} \vartheta}} \\
-\frac{\sin \theta \cos \theta \sin \vartheta \sin \phi}{\sqrt{\varepsilon^{2} \cos ^{2} \vartheta+\sin ^{2} \vartheta}}\end{array}$ \\
\hline $\begin{array}{l}(o \rightarrow p) \\
\text { et } \\
(p \rightarrow o)\end{array}$ & $o$ & $\begin{array}{l}\frac{\varepsilon \sin \theta \cos \theta \cos \vartheta \sin \phi}{\varepsilon^{2} \cos ^{2} \theta+\sin ^{2} \theta} \\
\sin \theta \cos \theta \cos \vartheta \sin \phi\end{array}$ \\
\hline$(e \rightarrow e)$ & $k$ & $\begin{array}{c}\frac{\sin ^{2} \vartheta}{\varepsilon^{2} \cos ^{2} \vartheta+\sin ^{2} \vartheta} \\
\varepsilon^{2} \frac{\sin ^{2} \vartheta \cos ^{2} \theta+\sin ^{2} \theta \cos ^{2} \vartheta+2 \sin \vartheta \cos \vartheta \sin \theta \cos \theta \cos \phi}{\left(\varepsilon^{2} \cos ^{2} \vartheta+\sin ^{2} \vartheta\right)\left(\varepsilon^{2} \cos ^{2} \theta+\sin ^{2} \theta\right)} \\
\frac{\varepsilon^{2} \cos ^{2} \theta \cos ^{2} \vartheta+\sin ^{2} \theta \sin ^{2} \vartheta-2 \varepsilon \sin \theta \cos \theta \sin \vartheta \cos \vartheta \cos \phi}{\varepsilon^{2} \cos ^{2} \vartheta+\sin ^{2} \vartheta}\end{array}$ \\
\hline$(e \rightarrow p)$ & $k$ & $\begin{array}{c}\frac{\sin \vartheta \cos \vartheta}{\sqrt{\varepsilon^{2} \cos ^{2} \vartheta+\sin ^{2} \vartheta}} \\
-\varepsilon \frac{\sin \vartheta \cos \vartheta\left(\varepsilon \cos ^{2} \theta-\sin ^{2} \theta\right)+\sin \theta \cos \theta \cos \phi\left(\varepsilon \cos ^{2} \vartheta-\sin ^{2} \vartheta\right)}{\left(\varepsilon^{2} \cos ^{2} \theta+\sin ^{2} \theta\right) \sqrt{\varepsilon^{2} \cos ^{2} \vartheta+\sin ^{2} \vartheta}} \\
\frac{\sin \theta \cos \theta \cos \phi\left(\varepsilon \cos ^{2} \vartheta-\sin ^{2} \vartheta\right)+\sin \vartheta \cos \vartheta\left(\varepsilon \cos ^{2} \theta-\sin ^{2} \theta\right)}{\sqrt{\varepsilon^{2} \cos ^{2} \vartheta+\sin ^{2} \vartheta}}\end{array}$ \\
\hline$(p \rightarrow p)$ & $o$ & $\begin{array}{c}\cos ^{2} \vartheta \\
\frac{\varepsilon^{2} \cos ^{2} \theta \cos ^{2} \vartheta+\sin ^{2} \theta \sin ^{2} \vartheta-2 \varepsilon \sin \theta \cos \theta \sin \vartheta \cos \vartheta \cos \phi}{\varepsilon^{2} \cos ^{2} \theta+\sin ^{2} \theta} \\
\sin ^{2} \vartheta \cos ^{2} \theta+\cos ^{2} \vartheta \sin ^{2} \theta+2 \sin \theta \cos \theta \sin \vartheta \cos \vartheta \cos \phi\end{array}$ \\
\hline
\end{tabular}


a)
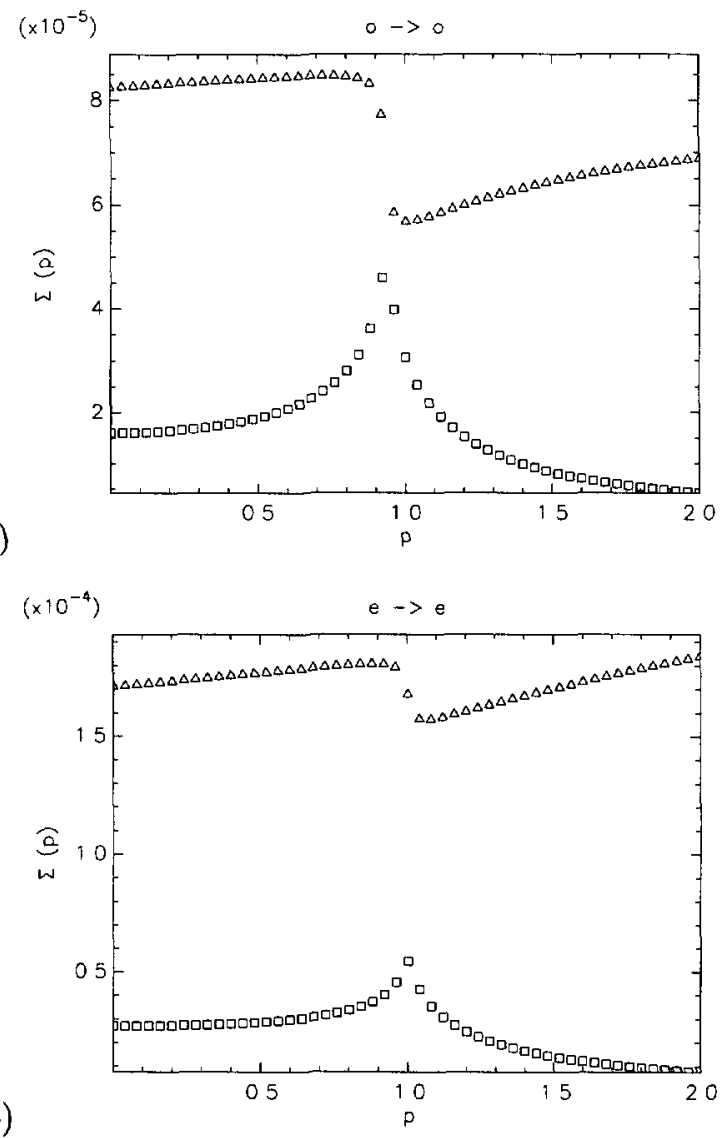

Fig. 1. - The mass operator as a function of the wave vector absolute value for the ordinary and extraordinary component. The curves are parametrized by the angle between the direction of propagation and the optical axis. In the represented graph $\theta=\pi / 2$. The triangles correspond to the real part of $\Sigma$, while the squares represent $-\operatorname{Im} \Sigma$.

3.1. Influence of Selection Rules. - The influence of long range fluctuations in nematic liquid crystals has already been discussed in a separate paper, using a scalar approximation [9]. We investigated the dependence of the mass operator on the absolute value of the wave vector $p$ in the first Born approximation. For the imaginary part of $\Sigma$ we observe a logarithmic divergence at $p=\omega / c$, if the correlation length is infinite. This divergence leads to an anomalous behavior of the spectral function. Its maximum splits into two peaks. This effect disappears in a self-consistent treatment of the mass operator.

The $p$-dependence of the mass operator is shown in Figure 1. The fact that the ordinary ray cannot keep its state of polarization after being scattered has an important consequence for the component $\Sigma_{o o}=\langle\mathbf{o}|\boldsymbol{\Sigma}| \mathbf{o}\rangle$ of the mass operator. Inside the diagram (11) no ordinary polarization can exist. $\Sigma_{o o}$ depends only on the extraordinary and longitudinal components of the unperturbed Green's function. Since the latter is a real valued quantity, the major contribution to the imaginary part of $\boldsymbol{\Sigma}$ comes from the shell of constant frequency of the extraordinary ray. As in the scalar approximation, we obtain a logarithmic divergence of $\operatorname{Im} \boldsymbol{\Sigma}$ if the correlation length is infinite. However, for the component $\Sigma_{o o}$ this divergence occurs at 

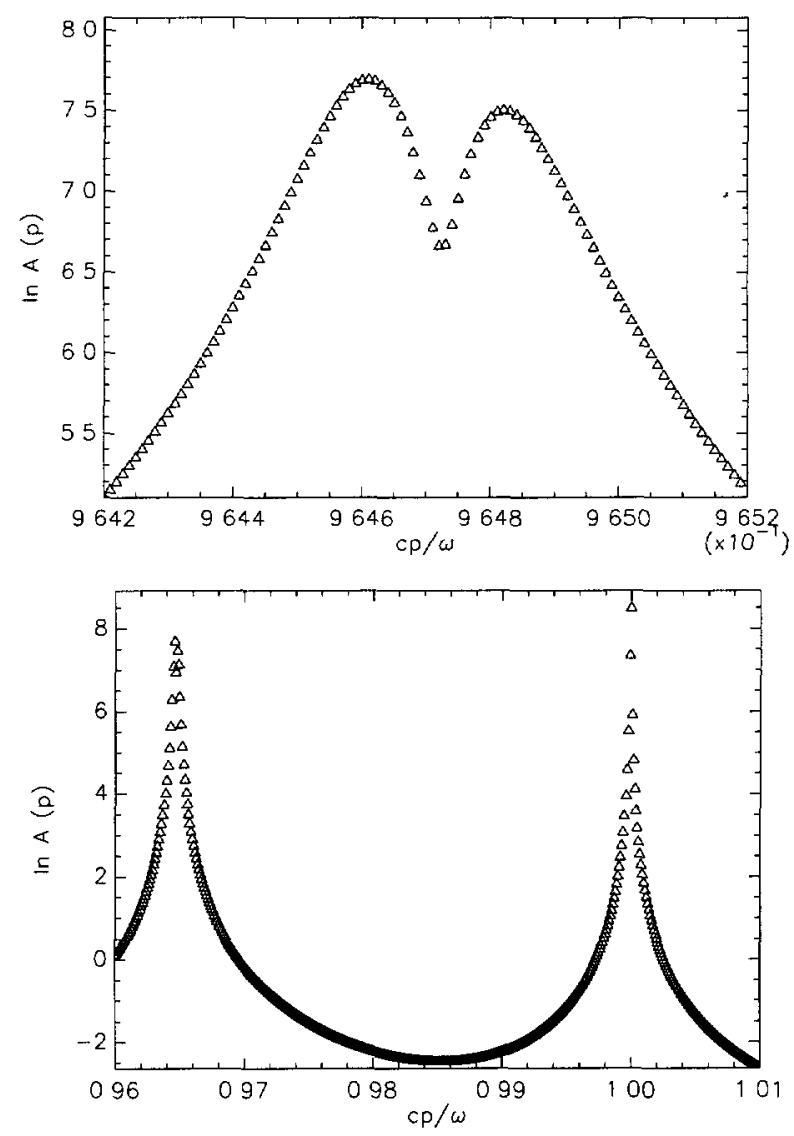

Fig. 2. - The spectral function (in arbitrary units). The bottom figure shows the ordinary (right) and extraordinary (left) excitation. The top figure shows that the extraordinary excitation is in fact split up into two in the first Born approximation made here. The ordinary excitation does not split up due to a selection rule.

$p=p_{e}$ and not at $p=p_{o}$. The spectral function is defined by the following relation:

$$
\begin{aligned}
A_{j}(\mathbf{p}, \omega) & =\frac{-2 \omega}{\pi} \operatorname{Im} \mathbf{G} \\
& =\frac{-2 \omega}{\pi} \frac{n_{\jmath}^{2} \cos ^{2} \delta_{\jmath} \operatorname{Im} \Sigma_{\jmath j}(\mathbf{p}, \omega)}{\left[\cos ^{2} \delta_{j}\left(\mathbf{p}_{\jmath}^{2}-p^{2}\right)-\operatorname{Re} \Sigma_{\jmath \jmath}(\mathbf{p}, \omega)\right]^{2}+\left[\operatorname{Im} \Sigma_{\jmath \jmath}(\mathbf{p}, \omega)\right]^{2}}
\end{aligned}
$$

The logarithmic divergence of the mass operator leads to a local minimum of the spectral function at $p=p_{e}$, but not to a splitting of the maximum which is located at $p=p_{o}$. Since we are mostly concerned with the value at $p=p_{o}$ (on shell), for instance if we apply the Boltzmann approximation in order to calculate the average intensity [8], a modification of the spectral function at $p=p_{e}$ has no consequences. The problem continues to exist only in the direction parallel to the optical axis because both polarizations obey the same dispersion law in this direction. The spectral function is shown in Figure 2 for an angle $\theta=\pi / 4$ between the direction of propagation and the optical axis. 
The extraordinary component $\Sigma_{e e}$ is not restricted by a selection rule. All modes of the Green's function $\mathbf{G}_{0}$ contribute to the diagram (11) and we observe a divergence of the imaginary part of the mass operator at $p=p_{e}$, the shell of constant frequency of the extraordinary ray. A splitting of the spectral function into two peaks can be observed. A similar effect obtained using a scalar approximation turned out to be absent, because a self-consistent treatment of the mass operator using the scalar approximation destroys the splitting [9]. A selfconsistent calculation taking into account the vector character of the field is far from being evident, because of the complexity of the analysis. It is not clear to us, whether the pathological double-peaked form of the spectral function is a physical effect or just an artifact of the calculation.

3.2. Propagating Versus Longitudinal Field. - Let us consider in detail the integral over the absolute value of the wave vector $k$ in relation (13). The imaginary part can easily be solved by Cauchy integration. In the real part of $\boldsymbol{\Sigma}$ we encounter a problem of convergence. The contribution proportional to the longitudinal component of $\mathbf{G}_{0}$ diverges for large values of $k$, i.e. for small distances $r$ in real space.

First, let us study the propagating part of the field. We have to solve an integral of the following type:

$$
\left(\frac{\omega}{c}\right)^{4} \Lambda \int_{0}^{\infty} \frac{k^{2} \mathrm{~d} k}{a k^{2}+b k+c} \frac{1}{k_{\jmath}^{2}-k^{2}+i 0^{+}}
$$

which does not confront us with any problems of convergence. Details of the rather tedious calculation can be found in Appendix A. The imaginary part of the mass operator is given by the sum of two integrals similar to (15), corresponding to the two propagation modes. Being a real valued function, the longitudinal component does not contribute to $\operatorname{Im} \Sigma$. We note that the result varies essentially as $\omega^{3}$, i.e. the ratio $\operatorname{Im} \Sigma / \omega^{2}$ vanishes in the limit $\omega \rightarrow 0$.

On the contrary, the longitudinal part of the Green's function contributes to the real part of $\boldsymbol{\Sigma}$. It leads to an integral of the following type:

$$
\left(\frac{\omega}{c}\right)^{2} \Lambda \int_{0}^{\infty} \frac{k^{2} \mathrm{~d} k}{a k^{2}+b k+c}
$$

which diverges in the ultra-violet regime even if after having done the angular integration. A physical interpretation of this divergence remains to be done.

In our case, the correlation function $\langle\delta \varepsilon(\mathbf{q}) \delta \varepsilon(0)\rangle$ in equation (8) has been obtained using the continuous medium theory of liquid crystals. The validity of this theory breaks down on length scales smaller than the size of the molecules $a_{0}$. Therefore, we cut off our interval of integration over $k$ at the value $1 / a_{0}$. Typically, $a_{0}$ is of the order of ten angstroms. Once renormalized, the real part of the mass operator can be calculated. In contrast to the imaginary part, the real part of $\Sigma$ varies like $\omega^{2} \Lambda / a_{0}$ and the ratio $\operatorname{Re} \Sigma / \omega^{2}$ remains finite in the limit $\omega \rightarrow 0$. In the static limit only the correction of the refractive index due to the longitudinal field survives, while all extinction due to scattering processes vanishes.

3.3. Renormalized Dispersion Laws. - Thermal fluctuations of the molecular orientation lead to a renormalization of the dispersion laws. In order to calculate the correction, we have to determine the zeros of the determinant of the denominator of the average Green's function (10),

$$
\operatorname{det}\left|\mathbf{G}_{0}^{-1}(\mathbf{p}, \omega)-\boldsymbol{\Sigma}(\mathbf{p}, \omega)\right|=0
$$


Expressed in the complete, but non-orthogonal basis $\{|\mathbf{o}\rangle,|\mathbf{e}\rangle$ and $|\mathbf{p}\rangle\}$, the mass operator takes the following form,

$$
\Sigma_{2 f}(\mathbf{p}, \omega)=\left(\begin{array}{ccc}
\Sigma_{o o} & 0 & 0 \\
0 & \Sigma_{e e} & \Sigma_{e p} \\
0 & \Sigma_{e p} & \Sigma_{p p}
\end{array}\right)
$$

This implies that the ordinary component does not couple with the extraordinary and longitudinal components. As a result, Fresnels law (17) decouples into two equations:

$$
\begin{aligned}
& 0=p_{o}^{2}-p^{2}-\Sigma_{o o}(\hat{\mathbf{p}}, \omega) \\
& 0=\left[\cos ^{2} \delta_{\jmath}\left(p_{e}^{2}-p^{2}\right)-\Sigma_{e e}(\hat{\mathbf{p}}, \omega)\right]\left[(\omega / c)^{2}\left\langle\mathbf{p}\left|\varepsilon_{0}\right| \mathbf{p}\right\rangle-\Sigma_{p p}(\hat{\mathbf{p}}, \omega)\right]-\Sigma_{e p}^{2}(\hat{\mathbf{p}}, \omega)
\end{aligned}
$$

In principle, $\Sigma(\mathbf{p}, \omega)$ is not only a function of the direction of $\mathbf{p}$ but also of the absolute value $p$. However, to first order in the perturbation series, it suffices to calculate $\Sigma$ for the non-perturbed values of $\omega$ and $\mathbf{p}$.

The angular dependence of the four matrix elements $\Sigma_{2 f}$ in (18) is shown in Figure 3. The absolute value of $\mathbf{p}$ is chosen on shell $\left(p=p_{e}\right.$ for the extraordinary ray, $p=p_{o}$ for the rest). Solid curves represent the real part of the mass operator, while dashed curves correspond to the imaginary part. The presence of thermal fluctuations influences the dispersion law of the ordinary ray in a crucial way, since the renormalized refractive index for the ordinary ray depends on the direction of propagation.

To first order in the coupling constant $\Lambda$, the renormalized dispersion laws are given by $p=p_{\jmath}^{(1)}$ where

$$
\begin{aligned}
& \operatorname{Re} p_{j}^{(1)}=p_{\jmath}-\frac{p_{\jmath}}{2} \frac{\operatorname{Re} \Sigma_{j \jmath}(\mathbf{p}, \omega)}{\left\langle\mathbf{j}\left|\varepsilon_{0}\right| \mathbf{j}\right\rangle}=p_{\jmath}-\frac{\operatorname{Re} \Sigma_{\jmath \jmath}(\mathbf{p}, \omega)}{2 p_{\jmath} \cos ^{2} \delta_{\jmath}} ; \\
& \operatorname{Im} p_{\jmath}^{(1)}=-\frac{p_{\jmath}}{2} \frac{\operatorname{Im} \Sigma_{\jmath \jmath}(\mathbf{p}, \omega)}{\left\langle\mathbf{j}\left|\varepsilon_{0}\right| \mathbf{j}\right\rangle}=-\frac{\operatorname{Im} \Sigma_{j j}(\mathbf{p}, \omega)}{2 p_{\jmath} \cos ^{2} \delta_{\jmath}}
\end{aligned}
$$

The eigenvectors change slightly for the extraordinary and longitudinal waves, whereas the ordinary ray is not affected. First order perturbation theory reveals that

$$
\begin{aligned}
& |\mathbf{o}\rangle \rightarrow|\mathbf{o}\rangle \\
& |\mathbf{e}\rangle \rightarrow|\mathbf{e}\rangle+C(\mathbf{p}, \omega)|\mathbf{p}\rangle \\
& |\mathbf{p}\rangle \rightarrow|\mathbf{p}\rangle-C(\mathbf{p}, \omega)|\mathbf{e}\rangle
\end{aligned}
$$

with

$$
C(\mathbf{p}, \omega)=\frac{1}{4} \frac{\Sigma_{e p}(\mathbf{p}, \omega)}{\left[(\omega / c)^{2}\left\langle\mathbf{p}\left|\varepsilon_{0}\right| \mathbf{p}\right\rangle-\cos ^{2} \delta_{e}\left(p_{e}^{2}-p^{2}\right)\right]}
$$

These vectors form a non-orthogonal basis.

3.4. Scattering Mean Free Path. - Multiple scattering of light leads to an attenuation of the wave. The extinction length, also called the scattering mean free path $l$, is related to the imaginary part of $\Sigma$, represented by the dashed curves in Figure 3 . It is defined by

$$
\ell_{j}^{-1}(\hat{\mathbf{p}}) \equiv-\frac{\operatorname{Im} \Sigma_{j \jmath}(\hat{\mathbf{p}}, \omega)}{\left(p_{j} \cos ^{2} \delta_{j}\right)}
$$



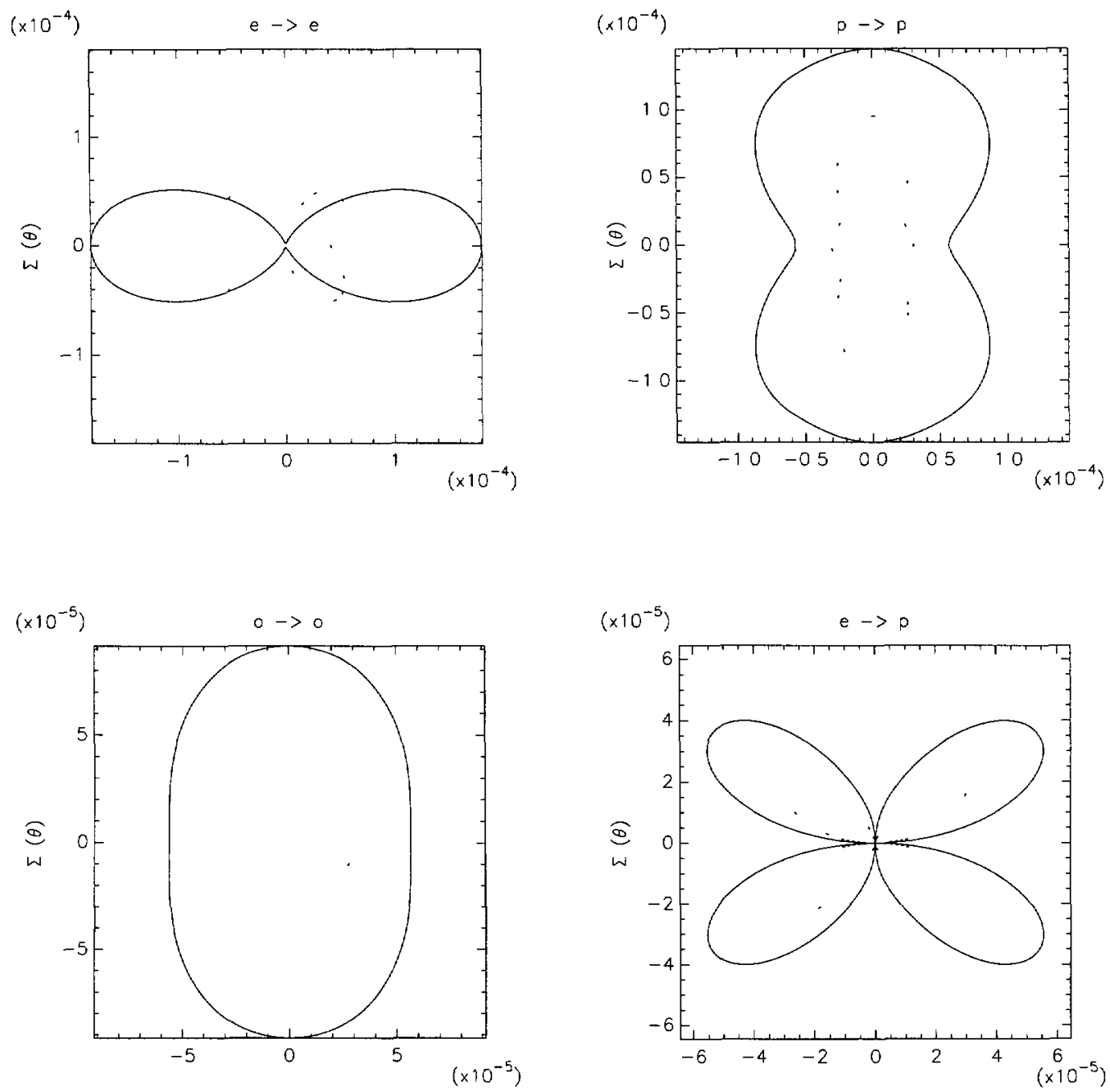

Fig. 3. - The mass operator as a function of the angle of propagation. Solid lines represent the real part, dashed lines the imaginary part of $\boldsymbol{\Sigma}$. Since the function is calculated on shell, $p$ varies with the angle for the extraordinary ray.

Note that the scattering mean free path depends on the direction of propagation as a result of the anisotropy of the medium. We are mostly interested in the components $\Sigma_{o o}$ and $\Sigma_{e e}$ since these determine the extinctions of the propagating matrix. The symmetry of the angular dependence of these two matrix elements is different, but we note that, independently of the sign of the anisotropy $\varepsilon_{\mathbf{a}}=\varepsilon_{\|}-\varepsilon_{\perp}$, the scattering lengths are maximal parallel to the optical axis. In that direction, the two polarization states $|\mathbf{e}\rangle$ and $|\mathbf{o}\rangle$ are degenerate (neglecting the correction $C$ in (23)) and we obtain the same numerical values for $l_{o / e}$. The longitudinal mode $|\mathbf{p}\rangle$ stays non propagative. 
With the notation $\mathbf{G}_{0}=\sum_{J} g_{j}|\mathbf{j}\rangle\langle\mathbf{j}|$ for the unperturbed Green's function, we find for the propagator $\langle\mathbf{G}\rangle$ of the average field:

$$
\begin{aligned}
\langle\mathbf{G}(\mathbf{p}, \omega)\rangle & =\left(\begin{array}{ccc}
\frac{1}{g_{o}^{-1}-\Sigma_{o o}(\mathbf{p}, \omega)} & 0 & 0 \\
0 & \frac{1}{g_{e}^{-1}-\Sigma_{e e}(\mathbf{p}, \omega)} & g_{e} g_{p} \Sigma_{e p}(\mathbf{p}, \omega) \\
0 & g_{e} g_{p} \Sigma_{e p}(\mathbf{p}, \omega) & \frac{1}{g_{p}^{-1}-\Sigma_{p p}(\mathbf{p}, \omega)}
\end{array}\right)+\mathcal{O}\left(\Sigma^{2}\right) \\
& \approx \sum_{\jmath=o}^{e} \frac{|\mathbf{p}\rangle\langle\mathbf{p}|}{\cos ^{2} \delta_{\jmath}\left(p_{\jmath}^{2}-p^{2}\right)-\Sigma_{\jmath \jmath}(\mathbf{p}, \omega)}+\frac{|\mathbf{j}\rangle\langle\mathbf{j}|}{(\omega / c)^{2}\left\langle\mathbf{p}\left|\varepsilon_{0}\right| \mathbf{p}\right\rangle-\Sigma_{p p}(\mathbf{p}, \omega)}
\end{aligned}
$$

The approximation consists of neglecting the non-diagonal terms which are directly proportional to the perturbation and which are therefore much smaller than the diagonal terms of $\langle\mathbf{G}\rangle$.

We have presented an analytical solution of Dyson's equation for the average electric field in ordered nematic liquid crystals when thermal fluctuations of the molecular orientation are present. As a result, we obtained the renormalized dispersion laws which can in principle be measured experimentally. Moreover, we determined the spectral function and the propagator of the average electric field.

\section{Monte-Carlo Simulation}

All calculations presented so far have been done for the average amplitude. Although the solution of Dyson's equation reveals various aspects concerning the long range of the fluctuations and the anisotropy of the medium, experimentalists are mainly interested in the average intensity. An analytical calculation in the diffusive regime using the Boltzmann approximation has been done and is published elsewhere [8]. In this paper. we present a different approach to the average intensity. We studied the radiative transfer in ordered nematic liquid crystals with a Monte-Carlo type simulation methor. This simulation program provides us with information about different quantities such as the shape and anisotropy of the coherent backscattering cone, the incoherent transmission coefficient, the time-of-flight distribution functions, the anisotropy of the diffusion coefficient and the depolarization of light. Expcrimentally, coherent backscattering has already been observed in oriented nenatic liquid crystals by Vithana et al. [11]. The advantage of a numerical simulation over an analytiral calculation is, that it permits to investigate the intermediate regime between single scattering and diffusion. However, this method does not allow to investigate effects related to the phase. since only the average intensity can be described correctly.

4.1. Algorithm. - Monte-Carlo simulations can be divided into two types: $a b$ initio simulations where no further assumptions are made other than the Hamiltonian, and non ab initio simulations where theory is incorporated in the simulation. Our Monte-Carlo simulation is of the second type. For example, we neglect the change in the refractive index due to the multiple scattering calculated above. This is possible since it was found to be of the order of $10^{-4}$ compared to 1 . Moreover, we do not take into account any cross-polarized terms, where two waves of different polarization interfere. These contributions disappear on the average because of the different dispersion laws that lead to a random phase difference between the two waves: $\left\langle E_{o} E_{e}^{*}\right\rangle=0$. Finally, we simulate the solution of the Bethe-Salpeter equation using 
Boltzmann's approximation and neglect any vertex corrections to the phase function and the mass operator.

In the Monte-Carlo simulation of multiple scattering processes, the diffusion of particles is described using a random walk model. The computer code contains a probabilistic kernel where in an iterative procedure four Markovian variables are determined at random, following known probability distributions. First, the distance between two scatterers is determined using an exponential distribution function (the Lambert-Beer law) with an attenuation length $l$, that results from the solution of Dyson's equation presented beforehand. The direction of propagation of the scattered wave is obtained using the conditional probability distributions described in detail in Appendix B. The scattering is highly anisotropic and takes place mostly in the forward direction. In contrast to existing Monte-Carlo simulations of multiple light scattering [3], an additional random variable exists for the transport problem in anisotropic media: the polarization. As mentioned before, no elliptical polarization can exist. Since the attenuation length depends on the polarization state of the wave, we cannot take the two rays into account at the same time, as is usually done for isotropic media. The existence of an additional random variable is an important difference between the vectorial approach and the scalar approximation [9] in anisotropic media, where no elliptical polarization can exist.

One fundamental problem of a Monte-Carlo simulation of multiple light scattering from long-range dielectric fluctuations is the concept of a "scatterer". A thermal fluctuation is not a scatterer in the sense a particle is. We deal with a collective excitation of an ensemble of molecules that induces a fluctuation in the refractive index. The range of this movement is described by the correlation length $\xi$. While the analytical calculation presented in the first part of this paper is valid for all correlation lengths, a numerical simulation can only be done if the scattering mean free path is much larger than the correlation length. Only in this case we may assume, that the far-field region of the precedent scattering process is reached before a new collision takes place. Fortunately, the condition $l / \xi \gg 1$ is fulfilled for typical parameters used during experiments. A magnetic field of 1 Tesla applied to MBBA corresponds to $\xi=3 \mu \mathrm{m}$, whereas the scattering mean free path is of the order of $100 \mu \mathrm{m}$.

The problem of multiple scattering in anisotropic media is a rather complicated one. Therefore, our computer code has to be more elaborate than in the isotropic case. We already mentioned one crucial complication: the polarization which implies, that the dispersion law of the extraordinary ray is anisotropic. We must also take into account that the group velocity of the extraordinary ray is not directed parallel to the phase velocity. The transport of energy after one scattering, though, will take place in the direction of the group velocity [16]. Moreover, the tensor character of the interaction leads to a dependence of all probability distributions on the polarization state and the direction of propagation. Finally, due to the anisotropy of the medium, all calculations have to be done in the laboratory frame, and not in a local frame.

We considered both an infinite medium and a slab of thickness $L$. In the case of a finite medium, two extreme orientations of the molecules are possible (see Fig. 4):

- geometry $(A)$ the optical axis is parallel to the surface of the slab;

- geometry $(B)$ the optical axis is oriented perpendicular to the surface.

Since we consider an oriented nematic liquid crystal, the optical axis defines one direction $\hat{\mathbf{z}}$ of the laboratory frame. In the case (A), a second direction $\hat{\mathbf{x}}$ is defined by the unit vector normal to the surface. We always considered normal incidence of a plane wave.

In order to avoid additional complications, we neglected refraction and internal reflections at the surface. Refraction could in principle be important for the backscattering cone, but its angular aperture turns out to be so small that all corrections are neglectable. The parameters used are summarized in Table II. The anisotropy parameter is defined as $\varepsilon=\varepsilon_{\perp} / \varepsilon_{\|}$. 


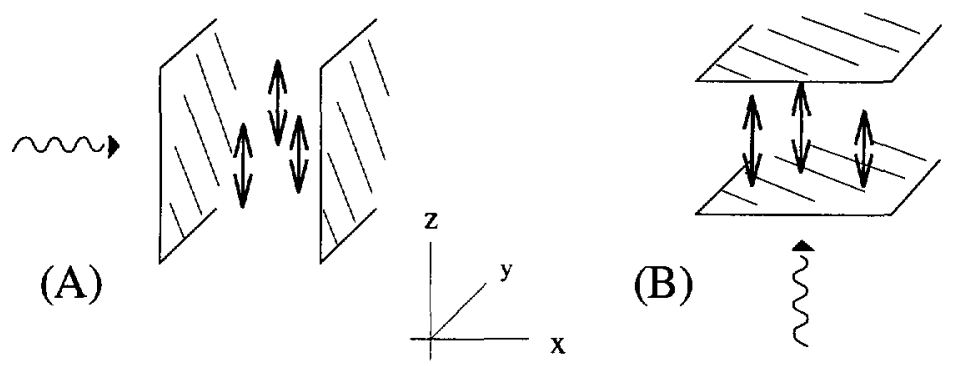

Fig. 4. - The two boundary conditions considered in this article. Geometry (A): The optical axis is oriented parallel to the surface of the slab. Geometry (B): The optical axis is oriented perpendicular to the surface. We consider normal incidence of a plane wave.

Table II. - The parameters used for the numerical simulations.

\begin{tabular}{|c|c|c|}
\hline$\varepsilon_{\perp}=4.7$ & $\chi_{\mathrm{a}}=1.23 \times 10^{-7} \mathrm{emu}$ & $B=1$ Tesla \\
\hline$K_{1}=K_{2}=6.7 \times 10^{-12} \mathrm{~N}$ & $K_{3}=8.4 \times 10^{-12} \mathrm{~N}$ & $T=300 \mathrm{~K}$ \\
\hline
\end{tabular}

\section{Numerical Results}

5.1. Coherent Backscattering. - In the middle of the eighties, weak localization of light was demonstrated both experimentally [1] and theoretically [2]. Since then, various experiments of the coherent backscattering cone have been done $[4,6,19-21]$. This constructive interference in the backscattering direction was also observed in nematic liquid crystals both in the oriented [11] and non-oriented phase [22]. In isotropic media, the shape of the cone very near the backscattering direction is triangular. From the slope of the triangle, the transport mean free path can be deduced. Experiments in ordered nematic liquid crystals [11] indicate a transport mean free path $l^{*}$ of the order of several millimeters. However, the angular resolution is not good enough to extract more quantitative results.

We simulated the coherent backscattering cone for different boundary conditions of the molecular orientation as well as for different planes of detection. Doing so, we were able to determine the anisotropy of the transport mean free path and the anisotropy of the cone shape. Once again, our simulation is not of an ab initio type. We neglected a priori the crosspolarized cones, where the incident polarization differs from the emergent polarization, $(o \rightarrow e)$ and $(e \rightarrow o)$. The absence of this contributions was already explained by Vithana et al. [11] for geometry (A). It is due to the different dispersion laws of the two rays. In the case of geometry (B), the distinction between ordinary and extraordinary rays is artificial, since the two dispersion laws coincide.

As a technical detail we mention that we used a method called "fractional photon method". This means that at each step the probability of the "photon" (here ment as a classical wave packet) to leave the slab either in transmission or reflection is determined. This probability is given by the product of the probability to travel over the distance between the actual scatterer and the border without being scattered and the probability to be scattered normal to the surface averaged over the possible angles of incidence (see Appendix B). The contribution of the "photon" to the transmitted or reflected intensity is weighted by this probability. Every "photon" is assumed to probe a possible realization of the medium and the superposition of the results leads to the ensemble averaged value. 
a)

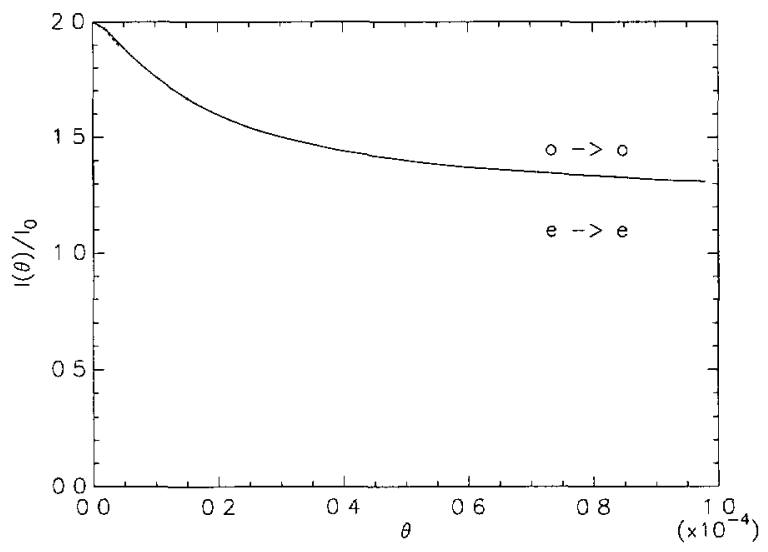

b)

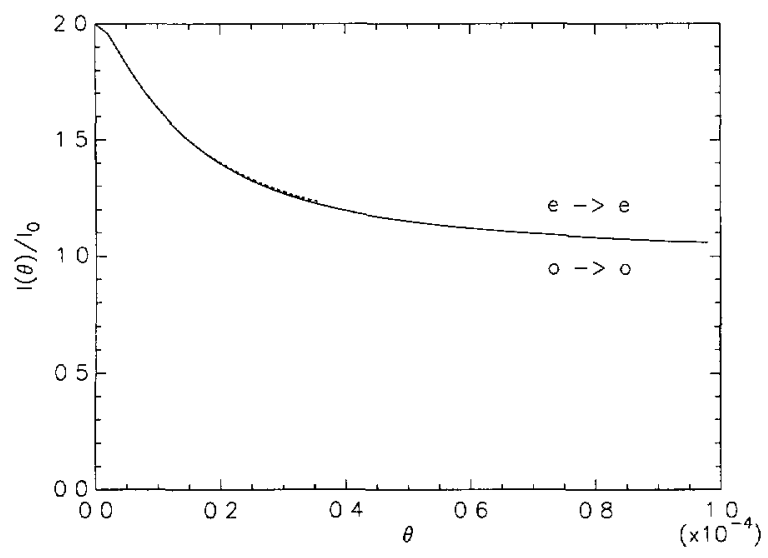

Fig. 5. - The coherent backscattering cone simulated for the ordinary polarization (solid line) and extraordinary polarization (dashed line) detected with respect to the optical axis. The relative anisotropy of the medium is $\varepsilon=1.3$. The figure a) was obtained for an orientation of the molecules parallel to the surface (A), while b) corresponds to a perpendicular orientation (B). The slab thickness is $2 \mathrm{~cm}$ and the angle is given in radians.

5.1.1. Line Shape of the Cone. - In Figure 5 we show the cone obtained for a thick slab. The total transmission, i.e. the transmission coefficient integrated over the half space, was found to be $T_{\text {total }}=6.4 \pm 0.2 \%$ for the upper figure and $T_{\text {total }}=8.9 \pm 0.2 \%$ for the lower one. The small rounding effect on top of the cone is due to these losses in transmission. Let us first consider the Figure 5b. In this case (B), the light is incident parallel to the optical axis, the only direction where the two rays are degenerated because their dispersion laws coincide. Therefore, no difference is observed between the cone detected with ordinary polarization and the cone with extraordinary polarization. Parallel to the optical axis, the two modes of linear polarization may combine to give an elliptical polarization, an effect which was neglected in the simulation, since it is a skin effect.

The situation is different for the geometry (A), where the light is incident normal to the optical axis. In this case the difference between the refractive indices is maximal. We will show that the difference of the slopes near the backscattering direction is due to this difference in the absolute value of the wave vectors. In an isotropic medium, the triangular shape of the 
Table III. - The transport mean free path as obtained by analyzing the line shape of the coherent backscattering cone. $l_{\mathrm{scal}}^{*}$ is the value obtained by a scalar approximation.

\begin{tabular}{|c||c|c|}
\hline & $\varepsilon=0.7$ & $\varepsilon=1.3$ \\
\hline$\left.\longrightarrow\right|^{\prime} / l_{1}$ & $l_{\perp}^{*}=0.44( \pm 0.02) l_{\text {scal }}^{*}$ & $l_{\perp}^{*}=0.50( \pm 0.04) l_{\text {scal }}^{*}$ \\
\hline$\frac{M l}{\uparrow}$ & $l_{\|}^{*}=0.69( \pm 0.07) l_{\text {scal }}^{*}$ & $l_{\|}^{*}=0.75( \pm 0.07) l_{\text {scal }}^{*}$ \\
\hline
\end{tabular}

cone is given theoretically by [2]:

$$
\frac{I(\theta)+I_{\text {inc }}}{I_{\text {inc }}}=2-2 \frac{(1+\zeta)^{2}}{1+2 \zeta} k l^{*} \theta .
$$

In order to interpret our results, we shall replace the wave vector $k$ in equation (27) by $k_{j}$, where we take into account the two different dispersion laws $(j=o, e)$. Moreover, we.have to replace $l^{*}$ by a quantity which depends on the geometry: $l_{\perp}^{*}$ for case (A) and $l_{\|}^{*}$ for case (B). Doing so, we indeed find that the triangular shape can be described by equation (27) with a transport mean free path that depends on the boundary conditions but not on the polarization. The results obtained for $l_{\perp / \|}^{*}$ are shown in Table III.

The length $l_{\text {scal }}^{*}$ corresponds to the transport mean free path as obtained using a scalar approximation, thus neglecting all polarization effects:

$$
l_{\mathrm{scal}}^{*}=\frac{8 \pi}{k_{o}^{2} \Lambda}=\frac{8 \pi \varepsilon_{\perp}^{2} K_{3}}{k_{o}^{2} \varepsilon_{\mathrm{a}}^{2} k_{\mathrm{B}} T} .
$$

For the parameters used in this paper, we obtain

$$
l_{\text {scal }}^{*}=771 \mu \mathrm{m} \text {. }
$$

We note that the transport mean free path deduced from our simulation is of the order of several hundreds of micrometers. It is smaller than the value obtained using a scalar approximation. This may reflect the fact that the polarization introduces an additional random variable in the problem of multiple scattering, which is not present in isotropic disordered media. An additional average is done. Therefore, the effect of the anisotropic scattering (mostly forward scattering) is diminished and the numerical value of $l^{*}$ is reduced.

Qualitatively, we can understand why the transport mean free path is larger in the direction parallel to the optical axis than perpendicular to it. The polarization of a wave propagating parallel to this direction is restricted to the plane perpendicular to $\mathbf{n}$. However, the refractive index in this plane is not affected by thermal fluctuations. Therefore, the wave will suffer only few scattering events and the transmission coefficient is larger than in the opposite case.

It is interesting to note that for the two boundary conditions studied in this paper, i.e. an orientation of the optical axis parallel or perpendicular to the surface normal, the enhancement factor will be strictly 2. This is due to the fact that no single scattering can occur in either geometry, the phase function being zero in both cases. For an oblique orientation of the molecules with respect to the boundaries, single scattering processes exist and the enhancement factor will be less than 2 . 
5.1.2. Anisotropy of the Cone. - Even in isotropic media the coherent backscattering cone is not isotropic, i.e. the lines of equal intensity are not spherical. The shape of the cone depends slightly on the plane in which it is detected. The anisotropy of the cone was investigated by Van der Mark et al. [23]. The authors find that only the wings of the backscattering cone in the linear polarized channel are modified when the detection plane is changed. In this case the anisotropy is due to low order scattering processes.

We studied the anisotropy of the cone in nematic liquid crystals for the two different boundary conditions (A) and (B) shown in Figure 4. In case (A), the optical axis is perpendicular to the surface normal, which coincides with the direction of the incident wave vector, $\mathbf{n} \perp \hat{\mathbf{p}}$. The cone can be detected either in the plane formed by those two vectors or in the plane perpendicular to the optical axis. As in the isotropic case, we expect an anisotropy of the cone. Situation (B) is different. In that case, the plane wave is incident parallel to the optical axis and the surface normal. No direction in the plane perpendicular to the optical axis can be distinguished. Therefore, we do not expect any anisotropy of the cone.

The Monte-Carlo simulation confirms these considerations. Indeed, for geometry (B) no anisotropy is observed. In Figure 6 the normalized reflected intensity is shown for the geometry (A). The upper figure was obtained for ordinary polarization ( $o \rightarrow o$ channel) while the lower one corresponds to extraordinary polarization $(e \rightarrow e$ channel), adopting a relative anisotropy of $\varepsilon=0.7$ and a slab thickness of about $3 \mathrm{~cm}$. We observe three principal results:

1. a change of the detection plane leaves the triangular top of the cone unchanged. Only the wings are modified;

2. the cone detected in the $o \rightarrow o$ channel is more affected than the one in the $e \rightarrow e$ channel;

3. the cone detected in the plane given by the surface normal and the optical axis is larger than the one obtained in the plane perpendicular to the optical axis.

The first point has also been observed by Van der Mark for Rayleigh and Mie scatterers in an isotropic medium. We conclude that only short paths with few scattering events are responsible for the anisotropy of the cone. We can also understand quantitatively why the cone detected in the plane given by the surface normal and the optical axis is larger than the cone obtained in the plane perpendicular to $\mathbf{n}$. The phase functions depend strongly on the angle of the incident and scattered waves vectors with respect to the optical axis. However, the choice of a principal axis of the system as direction of propagation is a very particular one. Several lower order scattering sequences are suppressed for this particular choice. Once the direction of propagation deviates from the principle axis, as is the case when the cone is detected in the plane parallel to the optical axis, the phase functions suffer less restrictions and lower order scattering events become more probable. This fact explains why the cone obtained in the plane parallel to $\mathbf{n}$ is found to be larger than the "perpendicular" one, since for the latter every direction perpendicular to the optical axis can be chosen as principle axis.

5.2. Transmission Coefficient. - In the precedent section we determined the transport mean free path by analyzing the line shape of the backscattering cone. An alternative approach to this quantity is given through the transmission coefficient $T$. The transmission coefficient used in this article is defined as the specific intensity transmitted through a slab, integrated over all possible orientations of the direction of detection with respect to the optical axis. Numerically, we counted the number of photons transmitted without regard to their direction of propagation. We studied the dependence of $T$ on the slab thickness for different boundary conditions (A) and (B) as well as for two different values of the anisotropy $\varepsilon=0.7$ and $\varepsilon=1.3$. The results are represented in Figure 7. The slab thickness is given in units of the scattering 
a)

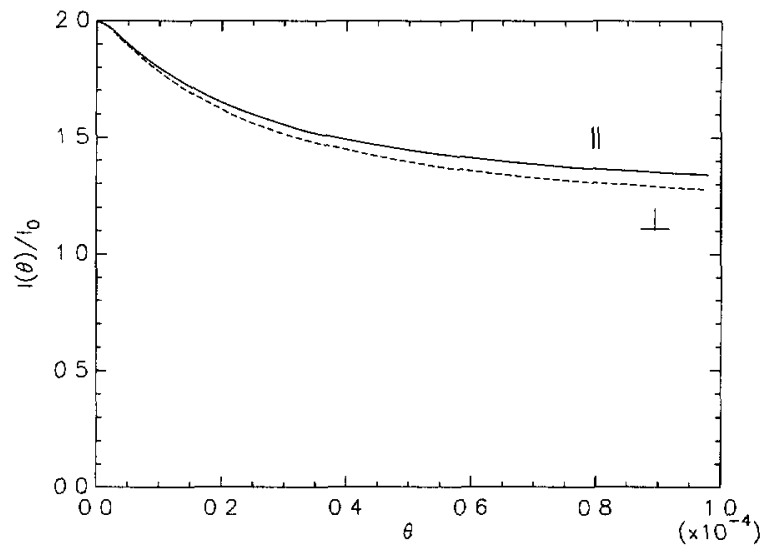

b)

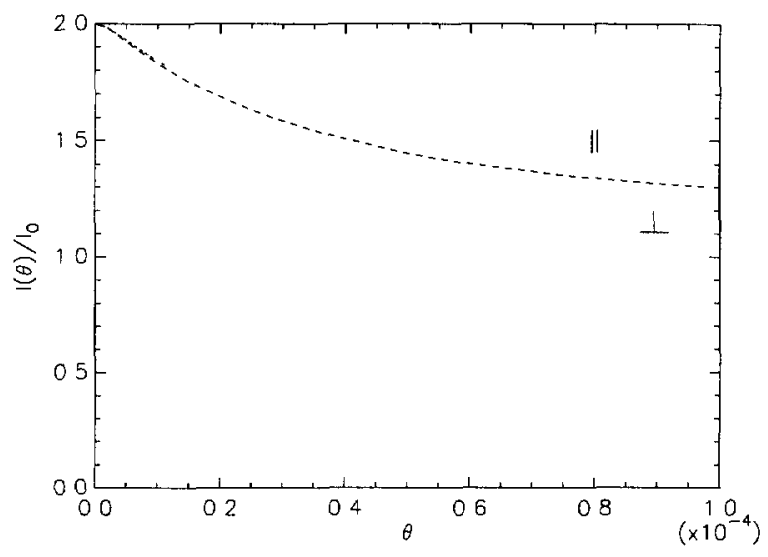

Fig. 6. - The coherent backscattering cone simulated for the two different a) ordinary and b) extraordinary polarizations. The optical axis is oriented parallel to the slab surface. Curves marked $\|$ are detected in the plane formed by the optical axis and the surface normal, curves marked $\perp$ are detected in the plane perpendicular to the optical axis. The relative anisotropy of the medium is $\varepsilon=0.7$ and the slab thickness values $3 \mathrm{~cm}$.

mean free path parallel to the optical axis, $l(\theta=0)$, which is the same for both polarization states $\left(l(\theta=0)=1.02 l_{\text {scal }}^{*}\right.$ for $\varepsilon=1.3$ and 0.8 for $\left.\varepsilon=0.7\right)$. The contribution of the ballistic photons was suppressed.

We note that the transmission coefficient is larger parallel to the optical axis (full symbols) than perpendicular to it (hollow symbols). For a given geometry it is smaller for a positive anisotropy ( $\varepsilon=1.3$, circles) than for the negative one $(\varepsilon=0.7$, diamonds). In the multiple scattering regime $(L \gg l(0))$ a linear relation between the slab thickness and the transport mean free path is established. This is analogous to the transmission through a disordered, but isotropic medium. For the isotropic case, the component of the specific intensity perpendicular to the slab normal is known [23] to be:

$$
T\left(\mu_{\mathrm{i}}, \mu_{\mathrm{s}}\right)=3 \mu_{\mathrm{i}} \mu_{\mathrm{s}} \frac{\left(\mu_{\mathrm{i}}+\zeta\right)\left(\mu_{\mathrm{s}}+\zeta\right)}{L / l^{*}+2 \zeta},
$$

where $\mu_{\mathrm{i}}=\cos \beta_{\mathrm{i}}$ describes the direction of incidence with respect to the slab normal, 


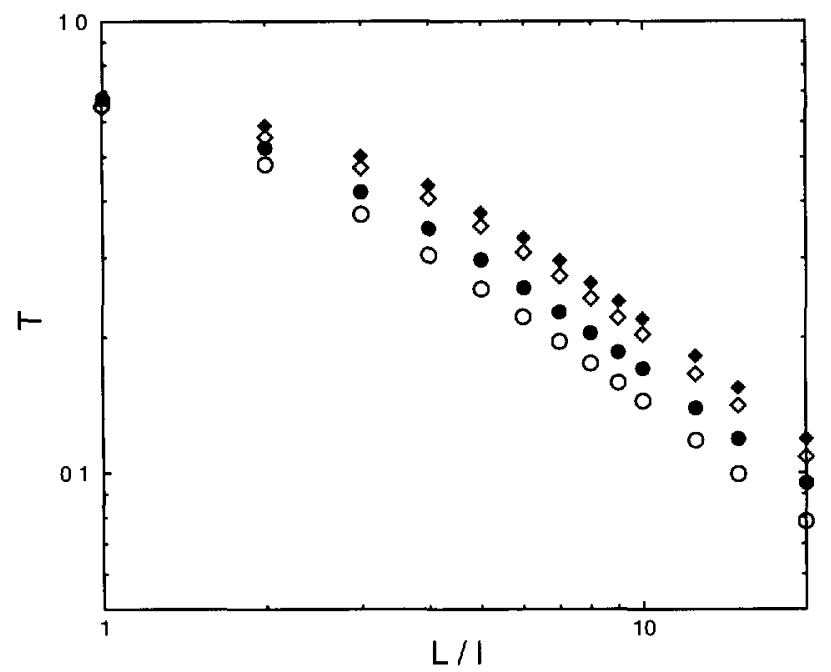

Fig. 7. - The transmission coefficient simulated for a positive anisotropy $(\varepsilon=1.3$ : circles $)$ and a negative anisotropy ( $\varepsilon=0.7$ : diamonds). Hollow symbols correspond to the geometry $(\mathrm{A})$ while full symbols correspond to geometry (B). A magnetic field of 1 Tesla was used to orient the molecules. (The error bars are of the size of the symbols.)

$\mu_{\mathrm{s}}=\cos \beta_{\mathrm{s}}$ refers to the direction of scattering, $l^{*}$ is the transport mean free path and $\zeta \approx 0.71$ is the parameter of the Milne problem. In our numerical simulation, $\mu_{\mathrm{i}}=1$ is fixed and we integrate over the angle of detection, which leads to:

$$
T_{\text {total }}=\int_{0}^{\pi} T\left(\mu_{\mathrm{i}}=1, \mu_{\mathrm{s}}\right) \mathrm{d} \mu_{\mathrm{s}}=2 \frac{(1+\zeta) l^{*}}{L+2 \zeta l^{*}}
$$

This result is valid in the diffusion approximation for an isotropic medium. (Note that the integration of (30) over all directions of incidence and detection leads to the rigorous result obtained from the radiative transfer equation [24].) In an isotropic medium, equation (31) allows us to determine the transport mean free path. $l^{*}$. We assume that relation (31) remains valid in anisotropic media for the two geometries if $l^{*}$ is replaced by a geometry-dependent value $l_{\perp}^{*}(\mathrm{~A})$ or $l_{\|}^{*}(\mathrm{~B})$. This hypothesis allows us to determine the transport mean free pathes $l_{\perp}^{*}$ and $l_{\|}^{*}$. However, this approach will give wrong results in the general case of an angle $\theta \neq 0, \pi / 2$ between the optical axis and the slab normal. The results, given in units of the transport mean free path obtained in a scalar approximation $\left(l_{\text {scal }}^{*} \approx 771 \mu \mathrm{m}\right)$, are summarised in Table IV.

Comparing Table III with Table IV, we conclude that the mean free path obtained via the transmission coefficient equals the one obtained by analyzing the line shape of the cone. Theoretically, this result is by no means trivial. It can be interpreted as an indication that the transport in the multiple scattering regime is in fact diffusive. We would like to emphasize that the onset of diffusion and the existence of a diffusion tensor is no longer obvious as soon as polarization and phase of the waves are explicitly taken into account. The diffusion pole is a subtle consequence of energy conservation in transport theory, in which case the losses of the coherent beam are exactly compensated by the scattering processes, including all polarization transitions and associated selection rules. 
Table IV. - The transport mean free path as obtained by analyzing the transmission coefficient. $l_{\text {scal }}^{*}$ is the value obtained by a scalar approximation.

\begin{tabular}{|c||c|c|}
\hline & $\varepsilon=0.7$ & $\varepsilon=1.3$ \\
\hline \hline$\longrightarrow \frac{\lambda}{1}$ & $l_{\perp}^{*}=0.44( \pm 0.02) l_{\mathrm{scal}}^{*}$ & $l_{\perp}^{*}=0.56( \pm 0.01) l_{\mathrm{scal}}^{*}$ \\
\hline$\frac{M \backslash}{\uparrow}$ & $l_{\|}^{*}=0.57( \pm 0.01) l_{\mathrm{scal}}^{*}$ & $l_{\|}^{*}=0.79( \pm 0.01) l_{\mathrm{scal}}^{*}$ \\
\hline
\end{tabular}

5.3. Time-of-Flight Distribution Functions. - Another quantity of interest that is closely related to the coherent backscattering cone is the path length distribution. In an isotropic medium, this distribution is equivalent to the time-of-flight distribution, which can be measured experimentally. However, in an anisotropic medium, the direct proportionality between $P(s)$ and $P(t)$ no longer holds because of the angular dependence of the velocity of the extraordinary ray. Since the analytical relation is unknown, we simulated both distribution functions in transmission as well as in reflection. No crucial difference between $P(s)$ and $P(t)$ was found within the error bars of our simulation.

We calculated the time-of-flight distribution function in reflection and transmission for different geometries and slab thicknesses. In order to analyze the curves, we supposed that the formulas obtained for an isotropic medium are still valid if we replace the transport mean free path and the diffusion coefficient by anisotropic quantities. This will only work for geometry (A) and (B), while in general a different formula has to be applied.

5.3.1. Reflection. - We are not interested in the exact formulas for the time-of-flight distribution function $P_{R}(t)$, but only in the asymptotic expression for long paths. In reflection from a disordered isotropic medium, this distribution corresponds to the probability of return in a random walk [25]. In the asymptotic diffusive regime of detection times much longer than the characteristic scattering time, $P_{R}(t)$ varies algebraically:

$$
P_{R}(t) \propto t^{-3 / 2}
$$

Our purpose was to confirm or invalidate this relation and to check explicitly the presence of normal long-range diffusion. In order to present the results of the Monte-Carlo simulation in Figure 8, a logarithmic representation is instructive. We studied different values for the slab thickness and the anisotropy. In all cases, the algebraic relation (32) was observed. We conclude that the transport is indeed diffusive like in isotropic media.

5.3.2. Transmission. - The asymptotic time-of-flight distribution function detected in transmission through a thick slab $P_{T}(t)$ can be obtained analytically using different methods [25-27]. In an disordered isotropic medium, it follows an exponential law, governed by the diffusion constant:

$$
P_{T}(t) \propto \mathrm{e}^{-t / \tau_{L}}=\mathrm{e}^{-\pi^{2} D t / L^{2}}
$$

This relation makes a semi-logarithmic representation useful (Fig. 9). Again supposing the validity of equation (33) in an anisotropic medium, we are able to deduce a value for the anisotropy of the diffusion coefficient, $D_{\perp} / D_{\|}$. A detailed analysis of the diffusion coefficient 
a)

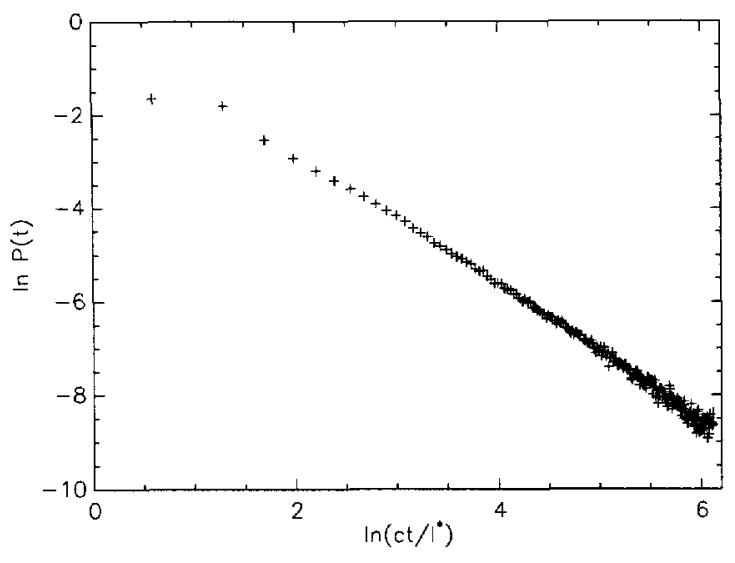

b)

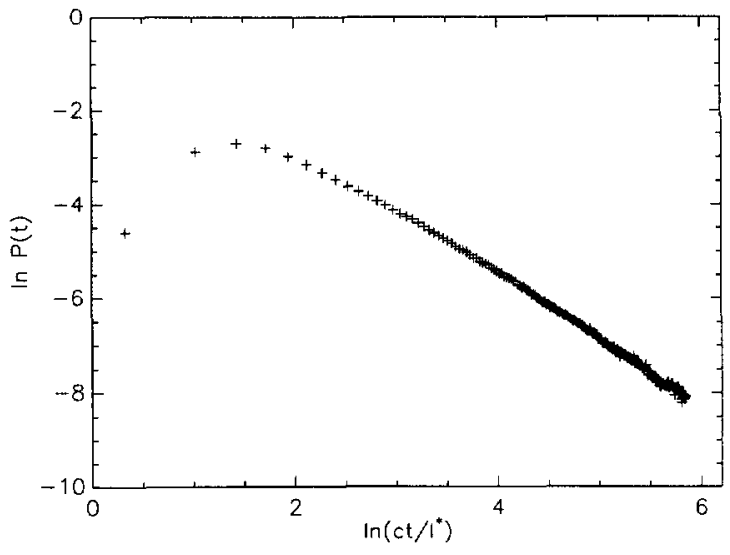

Fig. 8. - The time-of-flight distribution function in reflection from a slab of thickness $3 \mathrm{~cm}$ and an anisotropy $\varepsilon=0.7$ in a logarithmic representation. Where a) correponds to the geometry (A) and b) to the geometry (B).

can be found in reference [9]. At this stage of the analysis, we note that the relation

$$
D=\frac{1}{3} c l^{*}
$$

between the diffusion coefficient, the transport mean free path and the transport velocity known for isotropic media no longer holds in the anisotropic case. The values obtained for the ratio $D_{\perp} / c l_{\perp}^{*}$ and $D_{\|} / c l_{\|}^{*}$ are shown in Table $\mathrm{V}$. This result indicates that the velocity in the expression for the diffusion coefficient (34) is no longer a scalar, but depends on the angle between the direction of detection and the optical axis. This observation is confirmed by analytical calculations [9].

5.4. Polarization. - One well-known property of multiple scattering in isotropic disordered media is that initially polarized light will be completely depolarized $[3,29]$. We shall see that this is not true for anisotropic media. In order to study the polarization of light, we simulated two different quantities. 
a)

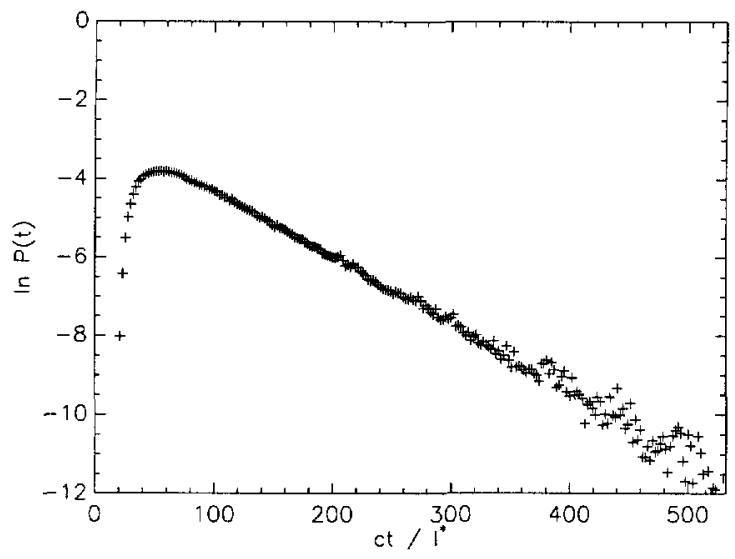

b)

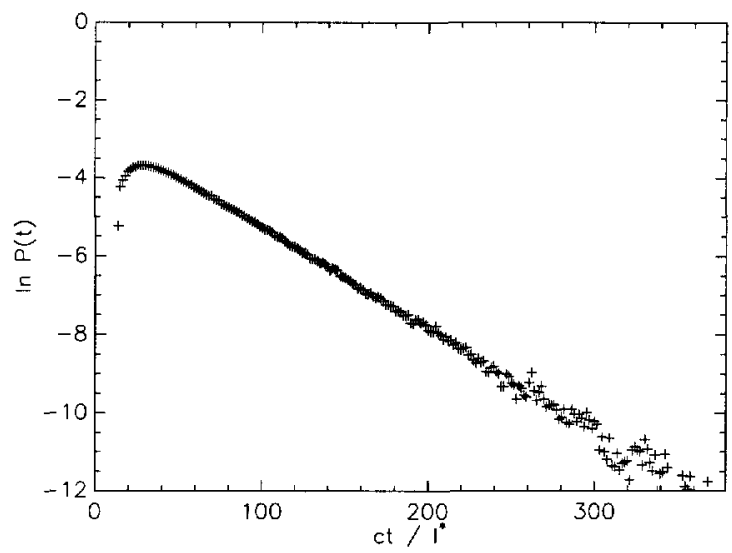

Fig. 9. - Time of-flight distribution function in transmission through a slab of thickness $6 \mathrm{~mm}$ and an anisotropy $\varepsilon=1.3$ in a half-logarithmic representation. Where a) correponds to the geometry (A) and $b)$ to the geometry (B).

Table V. - The linear relationship between the diffusion coefficient and the transport mean free path, known for isotropic media, no longer holds in the anisotropic case. (The velocity $c$ is only used in order to obtain a dimensionless quantity.)

\begin{tabular}{|c||c|c|}
\hline & $\varepsilon=0.7$ & $\varepsilon=1.3$ \\
\hline \hline$D_{\perp} / c l_{\perp}^{*}$ & $0.57( \pm 0.01)$ & $0.54( \pm 0.01)$ \\
\hline$D_{\|} / c l_{\|}^{*}$ & $0.37( \pm 0.01)$ & $0.43( \pm 0.02)$ \\
\hline
\end{tabular}

The "total" polarization:

in an infinite medium, photons are scattered several times. After a given time, we determine their polarization state without regard to the direction of propagation. The "total" polarization is defined as the ratio of the number of photons with ordinary polarization, normalized on the total number of photons. It corresponds to an angle average of the second quantity. 


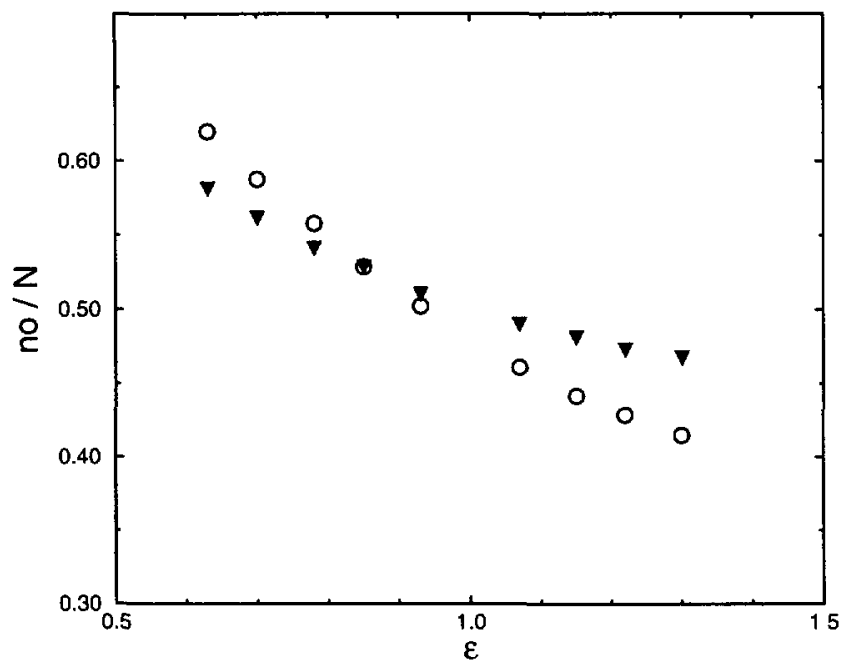

Fig. 10. - The "total" polarization as a function of dielectric anisotropy. (The error bars are of the size of the symbols.) The circles have been obtained by the Monte-Carlo simulation of multiple light scattering in an infinite medium. The triangles correspond to the result of an analytical calculation of the density of states, given by formula (36). Qualitatively, the two curves are in good agreement.

\section{The degree of polarization:}

this is given by the difference between the intensity detected in transmission as ordinary polarization and the one transmitted as extraordinary polarization, normalized on the total transmitted intensity:

$$
\eta_{p}=\frac{I_{o}-I_{e}}{I_{o}+I_{e}}
$$

Being only defined for the slab geometry, the value of $\eta_{p}$ is sensible to boundary conditions and depends on the direction of detection.

The incident light is assumed as being depolarized, i.e. containing 50\% ordinary and $50 \%$ extraordinary polarization.

In Figure 10, the total polarization is shown as a function of anisotropy. We note that the initially depolarized light is polarized in the multiple scattering regime. This rather astonishing result can be understood theoretically. We calculated the density of states available for ordinary and extraordinary polarization, given by the integral of the spectral function over all wave vectors. By the equipartition principle, the total polarization is expected to obey the following relation:

$$
\frac{N_{o}(\omega)}{N_{o}(\omega)+N_{e}(\omega)}=\frac{\int A_{o}(\mathbf{p}, \omega) \mathrm{d} \mathbf{p}}{\int A_{o}(\mathbf{p}, \omega) \mathrm{d} \mathbf{p}+\int A_{e}(\mathbf{p}, \omega) \mathrm{d} \mathbf{p}}
$$

where $A_{\jmath}(\mathbf{p}, \omega)$ is given by equation (15). To be consistent with our simulation, we have to neglect the real part of the mass operator, which corresponds to the change of the refractive index due to multiple scattering. We also neglected the existence of elliptical polarization, that can occur when the indices of refraction coincide. Analytically, this was taken into account by calculating $A_{o}$ and $A_{e}$ independently. 
In Figure 10 we see that the total polarization can at least qualitatively be explained by a difference in the available density of states for the two polarizations. We note that this difference is almost entirely due to the anisotropy of the host medium. Multiple scattering is only needed in order to reach equipartition.

Moreover, we determined the degree of polarization (35), which is defined for a finite sample size. As such, it is sensitive to the presence of the boundaries. In fact we observed, that the degree of polarization is highly influenced by the last scattering process which takes place in a given direction, in our case perpendicular to the surface and the optical axis. Because of the particular form of the phase functions, the degree of polarization was always found to be negative, indicating an excess of extraordinary polarization. Superposed to this skin effect, we find a decrease of the degree of polarization when the anisotropy is increased, as observed for the total polarization.

Finally, we mention a correlation effect found for the polarizations in transmission through a thick slab. In an infinite medium, no correlation between the first and last polarization state can exist. For us, this constituted a test of our program. However, we detected a residual correlation of about five percent even for a thick slab. This is also a skin effect, this time due to the first few collisions. As was said before, an initially depolarized light beam will be slightly polarized in a disordered anisotropic medium. Therefore, after a few collisions, an excess of one polarization state will be established. This excess is maintained all over the slab and results in a residual correlation between the incident and emergent polarization. In experiments, this effect should be observable.

\section{Conclusions}

In this paper we studied multiple scattering of light from long-range dielectric fluctuations in an anisotropic medium, taking polarization effects into account. Dyson's equation for the average field amplitude has been solved analytically. We generalized a previous work [12] concerning the angular dependence of the imaginary part of the mass operator. The dispersion law of the ordinary ray becomes anisotropic because of multiple scattering processes. Moreover, the contribution of the longitudinal wave leads to a divergent term that has to be renormalized, a fact that remains to be interpreted.

The logarithmic divergence of the imaginary part of the mass operator at the frequency shell, that was already observed within a scalar approximation [9], is also observed in the more rigorous tensor approach. However, the maximum for the ordinary component $\Sigma_{o o}$ is shifted because of a selection rule that applies to ordinary polarization. Therefore, the anomalous line shape of the spectral function, predicted by the scalar approximation is removed for the ordinary polarization. We determined the average Green's function and found a weak coupling between the longitudinal wave and the extraordinary ray, caused by multiple scattering.

A numerical approach was adopted in order to determine the average intensity. Using a Monte-Carlo simulation program, the coherent backscattering cone was calculated. We investigated the angular anisotropy of the cone qualitatively and concluded that only low order scattering processes are sensible to a change of the detection plane. The transport mean free path which depends on the direction of propagation relative to the optical axis was obtained quantitatively by analyzing the triangular top of the cone under the assumption that the theoretical line shape stays valid in anisotropic media. A similar analysis of the transmission coefficient offers an alternative way to obtain the geometry-dependent transport mean free path. The two methods lead to comparable results. This confirms the idea that the energy transport is in fact diffusive, since an analysis based an the diffusion approximation leads to consistent results. 
The asymptotic behavior of the time-of-flight distributions turn out to be well-described by the analytic expression obtained for an isotropic disordered medium taking an anisotropic diffusion coefficient into account. However, we note a crucial difference concerning the ratio of the diffusion coefficient and the transport mean free path. In an isotropic medium this ratio is constant, meaning that the transport velocity is a scalar constant. We find a dependence of the velocity on the angle of detection relative to the slab normal. This result has been obtained independently from an analytical solution of the Bethe-Salpeter equation [8].

Finally, we studied the polarization of the diffuse light. Contrary to the isotropic case, multiple scattering processes in anisotropic media do not depolarize the incident wave. The residual polarization depends on the anisotropy of the medium. This effect can be understood from a difference between the weight of the transport modes.

\section{Acknowledgments}

This work has been supported by the GDR "POAN". We thank Peter den Outer, Ralf Lenke, Georg Maret and Alex Martinez for discussions.

\section{Appendix A}

\section{Some Details of the Calculation of $\Sigma$}

In this appendix, we present some details of the calculation of the tensor mass operator $\mathbf{\Sigma}(\mathbf{p}, \omega)$. We have to solve the integral (13) taking into account the problems of convergence for the real part of $\boldsymbol{\Sigma}$ mentioned in Section 3.2. The imaginary part of $\boldsymbol{\Sigma}$ involves a delta-function and can easily be integrated. Since it does not possess a pole in the complex plane, the longitudinal part of the Green's function does not contribute to the imaginary part of $\boldsymbol{\Sigma}$. We find

$$
\begin{aligned}
\operatorname{Im} \Sigma(\mathbf{p}, \omega)= & \frac{-1}{16 \pi^{2}}\left(\frac{\omega}{c}\right)^{5} \Lambda \int_{k=p_{j}} \mathrm{~d} \Omega_{k_{3}} \sum_{j=o}^{e} \sum_{\alpha=1}^{2} \\
& \frac{n_{\jmath}}{\cos ^{2} \delta_{\jmath}} \frac{[|\mathbf{n}\rangle\langle\boldsymbol{\alpha} \mid \mathbf{j}\rangle+|\boldsymbol{\alpha}\rangle\langle\mathbf{n} \mid \mathbf{j}\rangle] \otimes[\langle\boldsymbol{\alpha} \mid \mathbf{j}\rangle\langle\mathbf{n}|+\langle\mathbf{n} \mid \mathbf{j}\rangle\langle\boldsymbol{\alpha}|]}{\left\langle\mathbf{q}_{j} \mid \mathbf{n}\right\rangle^{2}+A\left[q_{\jmath}^{2}-\left\langle\mathbf{q}_{\jmath} \mid \mathbf{n}\right\rangle^{2}\right]+1 / \xi^{2}}
\end{aligned}
$$

The remaining solid angle integral is taken over the surface of constant energy: $k=p_{j}=n_{3} \omega / c$. $q_{\jmath}$ denotes the quantity $\left|\mathbf{p}_{\jmath}-\mathbf{p}\right|$. We used the relation $\mathrm{d} \Omega_{k_{\jmath}}=\left(\cos \delta_{\jmath} / n_{\jmath}^{2}\right) \mathrm{d} \Omega_{r}$. The angular dependence of the numerator is shown in Table $\mathbf{I}$.

Obviously, the contributions of the "transverse" field components to the real part of $\boldsymbol{\Sigma}$ do not diverge either. The integral over the absolute value of $\mathbf{k}$ gives:

$$
\begin{aligned}
\mathcal{F}_{\jmath}= & \int_{0}^{\infty} \frac{k^{2} \mathrm{~d} k}{a k^{2}+b k+c} \frac{c^{2}}{\omega^{2}-\omega_{\jmath}^{2}(\mathbf{k})} \\
= & \frac{p_{j}}{4}\left\{\frac{1}{a p_{\jmath}^{2}-b p_{\jmath}+c}-\frac{1}{a p_{\jmath}^{2}+b p_{\jmath}+c}\right\} \ln \frac{c}{a p_{\jmath}^{2}} \\
& +\left\{\frac{p_{j}\left(2 a p_{\jmath}-b\right)}{4\left(a p_{\jmath}^{2}-b p_{J}+c\right)}+\frac{p_{j}\left(2 a p_{j}+b\right)}{4\left(a p_{j}^{2}+b p_{j}+c\right)}-1\right\} \\
& \times \frac{2}{\sqrt{4 a c-b^{2}}}\left(\frac{\pi}{2}-\arctan \frac{b}{\sqrt{4 a c-b^{2}}}\right) .
\end{aligned}
$$


We used the following notations:

$$
\begin{aligned}
& a=\cos ^{2} \theta+A \sin ^{2} \theta, \\
& b=-2 p(\cos \theta \cos \vartheta+A \sin \theta \sin \vartheta \cos \phi), \\
& c=p^{2}\left(\cos ^{2} \vartheta+A \sin ^{2} \vartheta\right)+1 / \xi^{2} .
\end{aligned}
$$

Here, $\theta$ is the angle between $\mathbf{k}$ and the optical axis $\mathbf{n}_{0}, \vartheta$ denotes the angle between $\mathbf{p}$ and $\mathbf{n}_{0}$ and $\phi$ corresponds to the angle between the projection of $\mathbf{k}$ and the projection $\mathbf{p}$ on the plane perpendicular to $\mathbf{n}_{0}$. The integrals over $\theta$ and $\phi$ can be solved numerically.

However, when treating the longitudinal contribution, we are confronted with an integral of the following type:

$$
\begin{aligned}
\int_{0}^{\infty} \frac{k^{2} \mathrm{~d} k}{a k^{2}+b k+c}= & {\left[\frac{k}{a}-\frac{b}{2 a^{2}} \ln \left(a k^{2}+b k+c\right)\right]_{0}^{\infty} } \\
& +\frac{b^{2}-2 a c}{2 a^{2}} \int_{0}^{\infty} \frac{\mathrm{d} k}{a k^{2}+b k+c}
\end{aligned}
$$

Only the third term is convergent:

$$
\mathcal{F}_{p}=\frac{b^{2}-2 a c}{a^{2} \sqrt{4 a c-b^{2}}}\left(\frac{\pi}{2}-\arctan \frac{b}{\sqrt{4 a c-b^{2}}}\right) .
$$

The second term vanishes after the integration of the solid angle. The first term remains present even after the angular integration. It has to be renormalized by limiting the integration interval by a cut-off at $k=1 / a_{0}$ which corresponds to the inverse of the size of a liquid crystal molecule.

The "divergent" part can now be integrated analytically:

$$
\begin{aligned}
\operatorname{Re} \Sigma_{\text {div }}(\mathbf{p}, \omega)= & \frac{(\omega / c)^{2}}{2 \pi^{2} a_{0}} \Lambda \int_{0}^{1} \frac{M_{i f} \mu^{2}+N_{i f}}{(1-A) \mu^{2}+A} \frac{\mathrm{d} \mu}{(\varepsilon-1) \mu^{2}+1} \\
= & \frac{(\omega / c)^{2}}{2 \pi^{2} a_{0}} \Lambda \frac{1}{A \varepsilon-1}\left[M_{\imath f}\left\{-\frac{\arctan \sqrt{\varepsilon-1}}{\sqrt{\varepsilon-1}}+\sqrt{\frac{A}{1-A}} \arctan \sqrt{\frac{1-A}{A}}\right\}\right. \\
& \left.+N_{\imath f}\left\{\sqrt{\varepsilon-1} \arctan \sqrt{\varepsilon-1}-\sqrt{\frac{1-A}{A}} \arctan \sqrt{\frac{1-A}{A}}\right\}\right]
\end{aligned}
$$

The angular coefficients $M_{i f}$ and $N_{\imath f}$ are given in Table VI. Note, that the anisotropy is entirely hidden in the relative parameters $A=K_{1} / K_{3}$ and $\varepsilon=\varepsilon_{\|} / \varepsilon_{\perp}$.

Finally, we obtain the following expression for the real part of the mass operator:

$$
\operatorname{Re} \Sigma(\mathbf{p}, \omega)=\frac{\varepsilon_{\mathrm{a}}^{2} k_{\mathrm{B}} T z^{4}}{(2 \pi)^{3} K} \int \mathrm{d} \Omega_{k}\left\{\sum_{j=o}^{e} \frac{1}{\cos ^{2} \delta_{\jmath}} \mathcal{F}_{\jmath} Q_{\imath f}^{j}+\frac{1}{z^{2}} \frac{1}{\langle k|\varepsilon| k\rangle} \mathcal{F}_{p} Q_{\imath f}^{p}\right\}+\operatorname{Re} \Sigma_{\text {div }}
$$

The integration over the solid angle was done numerically. We find, that the absolute value of $\operatorname{Re} \boldsymbol{\Sigma}$ is dominated by the contribution $\operatorname{Re} \Sigma_{\text {div }}$.

\section{Appendix B}

\section{Monte-Carlo Simulation}

We simulated multiple scattering processes numerically, using a Monte-Carlo simulation program. The principle part of this program is the probabilistic kernel, where the distance between 
Table VI. - The angular coefficients of equation (A.6) for the divergent part of $\operatorname{Re} \Sigma$.

\begin{tabular}{|c||c|c|}
\hline & $M_{i f}$ & $N_{\imath f}$ \\
\hline \hline$o \rightarrow o$ & 1 & 0 \\
\hline \multirow{2}{*}{$e \rightarrow e$} & $\frac{\varepsilon_{\|}^{2} \cos ^{2} \vartheta-\varepsilon_{\perp}^{2} \sin ^{2} \vartheta}{\varepsilon_{\|}^{2} \cos ^{2} \vartheta+\varepsilon_{\perp}^{2} \sin ^{2} \vartheta}$ & $\frac{\varepsilon_{\perp}^{2} \sin ^{2} \vartheta}{\varepsilon_{\|}^{2} \cos ^{2} \vartheta+\varepsilon_{\perp}^{2} \sin ^{2} \vartheta}$ \\
\hline$p \rightarrow p$ & $\sin ^{2} \vartheta-\cos ^{2} \vartheta$ & $\cos ^{2} \vartheta$ \\
\hline \multirow{2}{*}{$e \rightarrow p$} & $\frac{\left(\varepsilon_{\|}+\varepsilon_{\perp}\right) \sin \vartheta \cos \vartheta}{\sqrt{\varepsilon_{\|}^{2} \cos ^{2} \vartheta+\varepsilon_{\perp}^{2} \sin ^{2} \vartheta}}$ & $-\frac{\varepsilon_{\perp} \sin \vartheta \cos \vartheta}{\sqrt{\varepsilon_{\|}^{2} \cos ^{2} \vartheta+\varepsilon_{\perp}^{2} \sin ^{2} \vartheta}}$ \\
\hline
\end{tabular}

two scattering events, the direction of propagation of the scattered wave and the polarization after scattering is determined randomly, but following known probability functions. The knowledge of those functions is crucial for the quality of the simulation.

The probabilities of a wave with polarization $\alpha_{\mathrm{i}}$ and incident direction $\theta_{\mathrm{i}}, \phi_{\mathrm{i}}$ to be scattered in the direction $\theta_{\mathrm{s}}, \phi_{\mathrm{s}}$ with polarization $\alpha_{\mathrm{s}}$ is completely determined by the differential cross section [10] normalized on the total cross section:

$$
p\left(\alpha_{\mathrm{i}}, \theta_{\mathrm{i}} \mid \alpha_{\mathrm{s}}, \theta_{\mathrm{s}}, \Delta \phi\right)=\frac{\frac{\mathrm{d} \sigma}{\mathrm{d} \Omega}\left(\alpha_{\mathrm{i}}, \theta_{\mathrm{i}} \mid \alpha_{\mathrm{s}}, \theta_{\mathrm{s}}, \Delta \phi\right)}{\sum_{\alpha_{\mathrm{s}}} \int \frac{\mathrm{d} \sigma}{\mathrm{d} \Omega_{k}}\left(\alpha_{\mathrm{i}}, \theta_{\mathrm{i}} \mid \alpha_{\mathrm{s}}, \theta_{\mathrm{s}}, \Delta \phi\right) \mathrm{d} \cos \theta_{\mathrm{s}} \mathrm{d} \phi_{\mathrm{s}}}
$$

While this probability depends in a complex way on the direction of incidence and scattering with respect to the optical axis, only the relative polar angle $\Delta \phi=\phi_{\mathrm{s}}-\phi_{\mathrm{i}}$ enters in equation (B.1). It can be parametrized in the following way:

$$
p\left(\alpha_{\mathrm{i}}, \theta_{\mathrm{i}} \mid \alpha_{\mathrm{s}}, \theta_{\mathrm{s}}, \Delta \phi\right)=\Pi\left(\alpha_{\mathrm{i}}, \theta_{\mathrm{i}} \mid \alpha_{\mathrm{s}}\right) \mathcal{P}\left(\alpha_{\mathrm{i}}, \theta_{\mathrm{i}} ; \alpha_{\mathrm{s}} \mid \theta_{\mathrm{s}}\right) P\left(\alpha_{\mathrm{i}}, \theta_{\mathrm{i}} ; \alpha_{\mathrm{s}}, \theta_{\mathrm{s}} \mid \Delta \phi\right) .
$$

The different terms have the following physical meanings.

- The conditional probability of the polarization $\alpha_{\mathbf{s}}$ :

$$
\Pi\left(\alpha_{1}, \theta_{\mathrm{i}} \mid \alpha_{\mathrm{s}}\right)=\frac{\int \frac{\mathrm{d} \sigma}{\mathrm{d} \Omega_{k}}\left(\alpha_{\mathrm{i}}, \theta_{\mathrm{i}} \mid \alpha_{\mathrm{s}}, \theta_{\mathrm{s}}, \Delta \phi\right) \mathrm{d} \cos \theta_{\mathrm{s}} \mathrm{d} \phi_{\mathrm{s}}}{\sum_{\alpha_{\mathrm{s}}} \int \frac{\mathrm{d} \sigma}{\mathrm{d} \Omega_{k}}\left(\alpha_{\mathrm{i}}, \theta_{\mathrm{i}} \mid \alpha_{\mathrm{s}}, \theta_{\mathrm{s}}, \Delta \phi\right) \mathrm{d} \cos \theta_{\mathrm{s}} \mathrm{d} \phi_{\mathrm{s}}}
$$

Knowing the polarization $\alpha_{1}$ and the angle $\theta_{i}$ between the direction of propagation of the incident wave and the optical axis, $\Pi\left(\alpha_{i}, \theta_{\mathbf{i}} \mid \alpha_{\mathrm{s}}\right)$ enables us to determine the polarization $\alpha_{\mathrm{s}}$ of the scattered wave. 
- The conditional probability of the angle of propagation $\theta_{\mathrm{s}}$ relative to the optical axis:

$$
\mathcal{P}\left(\alpha_{\mathrm{i}}, \theta_{\mathrm{i}} ; \alpha_{\mathrm{s}} \mid \theta_{\mathrm{s}}\right)=\frac{\int \frac{\mathrm{d} \sigma}{\mathrm{d} \Omega_{k}}\left(\alpha_{\mathrm{i}}, \theta_{\mathrm{i}} \mid \alpha_{\mathrm{s}}, \theta_{\mathrm{s}}, \Delta \phi\right) \mathrm{d} \phi_{\mathrm{s}}}{\int \frac{\mathrm{d} \sigma}{\mathrm{d} \Omega_{k}}\left(\alpha_{\mathrm{i}}, \theta_{\mathrm{i}} \mid \alpha_{\mathrm{s}}, \theta_{\mathrm{s}}, \Delta \phi\right) \mathrm{d} \cos \theta_{\mathrm{s}} \mathrm{d} \phi_{\mathrm{s}}}
$$

Once the polarization state of the scattered wave is known, the angle between the scattered wave vector and the optical axis can be obtained.

- The conditional probability of the relative polar angle $\Delta \phi$ :

$$
P\left(\alpha_{\mathrm{i}}, \theta_{\mathrm{i}} ; \alpha_{\mathrm{s}}, \theta_{\mathrm{s}} \mid \Delta \phi\right)=\frac{\frac{\mathrm{d} \sigma}{\mathrm{d} \Omega_{k}}\left(\alpha_{\mathrm{i}}, \theta_{\mathrm{i}} \mid \alpha_{\mathrm{s}}, \theta_{\mathrm{s}}, \Delta \phi\right)}{\int \frac{\mathrm{d} \sigma}{\mathrm{d} \Omega_{k}}\left(\alpha_{\mathrm{i}}, \theta_{\mathrm{i}} \mid \alpha_{\mathrm{s}}, \theta_{\mathrm{s}}, \Delta \phi\right) \mathrm{d} \phi_{\mathrm{s}}}
$$

Moreover, we need the step length distribution $P(r)$. We assumed an exponential distribution, i.e. the Lambert-Beer law, with the scattering mean free path $l_{\alpha}\left(\theta_{\mathrm{i}}\right)$ as characteristic length. This is an approximation since we know that the step length distribution decreases slightly faster than an exponential [12].

Like the conditional probabilities, the scattering mean free path, obtained from the solution of Dyson's equation, is parametrized by $\theta_{\mathrm{i}}$ and $\alpha_{\mathrm{i}}$. In fact, since $l_{\alpha}\left(\theta_{\mathrm{i}}\right)$ depends on the polarization, we cannot follow the ordinary and extraordinary ray at the same time. Instead of superposing the two linear polarizations, we choose one polarization state which may change at each collision. Therefore, we deal with four random variables.

We generated the azimuthal angle by the cumulative function $[3,28]$, which is less time consuming in computing time than the rejection method. The relative polar angle can be obtained analytically for crossed polarization channels $o \rightarrow e, e \rightarrow o$, but an approximation was made for the $e \rightarrow e$ channel. This approximation underestimates slightly the anisotropy of the scattering process.

\section{References}

[1] van Albada M.P. and Lagendijk A., Phys. Rev. Lett. 55 (1985) 2692; Wolf P.E. and Maret G., Phys. Rev. Lett. 55 (1985) 2696.

[2] Akkermans E. and Maynard R., J. Phys. France Lett. 46 (1985) L-1045; Akkermans E., Wolf P.E. and Maynard R., Phys. Rev. Lett. 56 (1986) 1471.

[3] Martinez A.S. and Maynard R., Phys. Rev. B 50 (1994) 3714.

[4] Lenke R. and Maret G., to be published.

[5] van Tiggelen B.A., Phys. Rev. Lett 75 (1995) 422; Rikken G.L.J.A. and van Tiggelen B.A., Nature 381 (1996) 54.

[6] Wiersma D.S., van Albada M.P., van Tiggelen B.A. and Lagendijk A., Phys. Rev. Lett. 21 (1995) 4193.

[7] Heiderich A., Maynard R. and van Tiggelen B.A., Opt. Comm. 115 (1995) 392.

[8] van Tiggelen B.A., Maynard R. and Heiderich A., Phys. Rev. Lett. 77 (1996) 639.

[9] van Tiggelen B.A., Heiderich A. and Maynard R., to appear in Mol. Cryst. \& Liq. Cryst.

[10] Langevin D., Solid State Commun. 14 (1974) 435. 
[11] Vithana H.K.M., Asfaw L. and Johnson D.L., Phys. Rev. Lett. 70 (1993) 3561.

[12] Kuz'min V.L., Romanov V.P. and Zubkov L.A., Phys. Rep. 248 (1994) 71.

[13] Stark H. and Lubensky T.C., Phys. Rev. Lett. 77 (1996) 2229.

[14] Kao M.H., Jester K.A., Yodh A.G. and Collins P.J., Phys. Rev. Let. 77 (1996) 2233.

[15] Born M. and Wolf E., Principles of Optics (Pergamon Press, London, New York, Paris, Los Angeles, 1959).

[16] Landau L.D., Lifshitz E.M. and Piteavskii L.P., Electrodynamics of Continuous Media (Pergamon Press, Oxford, 1984).

[17] de Gennes P.G., The Physics of Liquid Crystals (Clarendon Press, Oxford, 1974).

[18] Chandrasekhar S., Liquid Crystals, 2nd ed. (Cambridge University Press, Cambridge, 1992).

[19] van Albada M.P. and Lagendijk A., Phys. Rev. B 36 (1987) 2353.

[20] Wolf P.E., Maret G., Akkermans E. and Maynard R., J. Phys. France 49 (1988) 63.

[21] Bayer G. and Niederdränk T., Phys. Rev. Lett. 70 (1993) 3884.

[22] Vlasov D.V., Zubkov L.A., Orekhova N.V., Romanov V.P., JETP Lett. 48 (1988) 91; [Pis'ma Zh. Eksp. Teor. Fiz. 48 (1988) 86].

[23] van der Mark M.B., van Albada M.P. and Lagendijk A., Phys. Rev. B 37 (1988) 3575.

[24] van de Hulst H.C., Multiple Scattering of Light (Academic Press, New York, 1980).

[25] Feller W., An Introduction to Probability Theory and its Applications (John Wiley \& Sons, Inc., Singapore, 3th ed., 1968).

[26] Crank J., The Mathematics of Diffusion, 2nd ed., Oxford Science Publications (Claredon Press, Oxford, 1975).

[27] Pine D.J., Weitz D.A., Zhu J.X. and Herbolzheimer E., J. Phys. France 51 (1990) 2101.

[28] Butcher E.A., Appl. Opt. 12 (1973) 2391; Kunkel K.E. and Weinman J.A., J. Atmos. Sciences 33 (1976) 1772.

[29] MacKintosh F.C., Zhu J.X., Pine D.J. and Weitz W.A., Phys. Rev. B 40 (1989) 9342. 